\title{
əComparing Adaptive Prior and Posterior Inflation for Ensemble Filters Using an Atmospheric General Circulation Model
}

\author{
Mohamad El Gharamti, Kevin Raeder, And JefFrey Anderson \\ National Center for Atmospheric Research, Boulder, Colorado \\ XUGUANG WANG \\ University of Oklahoma, Norman, Oklahoma
}

(Manuscript received 9 November 2018, in final form 8 March 2019)

\begin{abstract}
Sampling errors and model errors are major drawbacks from which ensemble Kalman filters suffer. Sampling errors arise because of the use of a limited ensemble size, while model errors are deficiencies in the dynamics and underlying parameterizations that may yield biases in the model's prediction. In this study, we propose a new time-adaptive posterior inflation algorithm in which the analyzed ensemble anomalies are locally inflated. The proposed inflation strategy is computationally efficient and is aimed at restoring enough spread in the analysis ensemble after assimilating the observations. The performance of this scheme is tested against the relaxation to prior spread (RTPS) and adaptive prior inflation. For this purpose, two model are used: the three-variable Lorenz 63 system and the Community Atmosphere Model (CAM). In CAM, global refractivity, temperature, and wind observations from several sources are incorporated to perform a set of assimilation experiments using the Data Assimilation Research Testbed (DART). The proposed scheme is shown to yield better quality forecasts than the RTPS. Assimilation results further suggest that when model errors are small, both prior and posterior inflation are able to mitigate sampling errors with a slight advantage to posterior inflation. When large model errors, such as wind and temperature biases, are present, prior inflation is shown to be more accurate than posterior inflation. Densely observed regions as in the Northern Hemisphere present numerous challenges to the posterior inflation algorithm. A compelling enhancement to the performance of the filter is achieved by combining both adaptive inflation schemes.
\end{abstract}

\section{Introduction}

Ensemble Kalman filtering techniques consist of successive forecast and analysis steps. During the forecast, a set of model realizations is advanced forward in time using the dynamical model. At the time of the analysis, available observations are assimilated using a linear Kalman-type update yielding a reduced prior ensemble uncertainty (Evensen 2003). Although proven successful in various Earth system applications, the ensemble Kalman filter (EnKF) might be subject to a number of errors and drawbacks. Depending on the severity of the

¿ Denotes content that is immediately available upon publication as open access.

Corresponding author: Mohamad El Gharamti, gharamti@ ucar.edu underlying case, these errors may yield a complete failure of the filtering procedure.

Such errors or deficiencies can be associated with both the forecast and the analysis steps. Model errors and sampling errors are two major factors that affect the performance of the filter at the forecast level (Furrer and Bengtsson 2007). Sampling error, in both the mean and the variance of the ensemble, results from using a limited ensemble size and thus the true probability distribution of the state may not be well represented. After a few assimilation cycles, sampling error may cause a strong underestimation of the true system's variance (Houtekamer and Mitchell 1998). In the presence of model bias, the model simulation produces ensemble predictions that are typically far from the true environment. In many cases, the big discrepancy between the model's prediction and the observations may lead to an ensemble collapse. During the analysis step, sampling and model errors persist and combine to yield 
additional inaccuracies while regressing state increments to the forecast ensemble (Anderson 2012). Systematic errors in the forward operators in addition to observational and representativity errors may produce incorrect observation increments, further contributing to deficiencies in the analysis step (Hodyss and Nichols 2015).

One way of mitigating the issue of variance underestimation, caused by sampling errors, is through prior covariance inflation (Wang and Bishop 2003, and references therein). The idea is that after integrating the ensemble members forward in time, the ensemble anomalies are inflated by a factor larger than 1 (Anderson and Anderson 1999). This increases the value of the second moment of the state's distribution in the hope that it can now match the, unknown, theoretical one (Pham 2001). Prior inflation has been successfully tested and applied in various Earth system data assimilation (DA) applications (e.g., Raeder et al. 2012; Hoteit et al. 2013). Anderson (2009, hereafter A09), proposed a spatially and temporally varying adaptive prior inflation scheme. The algorithm is based on Bayes's rule such that the inflation values are updated, at each observation time, locally in space by multiplying a Gaussian density by some nonGaussian likelihood function. Miyoshi (2011) proposed a fully Gaussian approximation to Anderson's scheme in which the likelihood is assumed normal and its moments are given by the maximum likelihood and central limit theorem. Recently, El Gharamti (2018, hereafter E18) introduced two enhancements to Anderson's inflation algorithm. In essence, the inflation prior distribution is represented by an inverse-gamma distribution and a positive correction term is added to the mode of the inflation likelihood. Other forms of inflation exist and the reader may refer to the recent studies of E18 and Raanes et al. (2019) for an extensive review.

Although prior covariance inflation was introduced as a practical solution to tackle sampling errors, studies have shown that it can also be used to mitigate for other deficiencies such as model errors. For instance, Dowell et al. (2004) and Aksoy et al. (2009) used an inflation factor of 1.05 to address different model errors in convective-scale atmospheric DA experiments. Other studies, such as Whitaker et al. (2008), suggested that additive inflation (e.g., Houtekamer et al. 2009) can efficiently help treat unrepresented model errors. Parameterizing a model error covariance could enhance the performance in the presence of model biases or poorly represented dynamics. This, however, is a hard task to achieve given the large size of the atmospheric state and our poor knowledge of various model related quantities such as the model's input, physical parameters and forcing.
If sampling and model errors are not resolved during the forecast, would inflating the analysis ensemble help reduce their negative impacts? Techniques such as the observation-dependent posterior inflation algorithm of Hodyss et al. (2016) and the relaxation to prior perturbations (Zhang et al. 2004; RTPP) or spread (Whitaker and Hamill 2012; RTPS) have shown strong potential at handling such filtering errors. Here, we propose a new, fully Bayesian, posterior inflation algorithm. The algorithm uses ideas from A09 and further applies the enhancements presented in E18. The derivation of the new scheme is slightly different than the adaptive prior inflation and it features an observation-impact removal strategy as an efficient way to compute the posterior inflation distribution. An illustrative example, using a small toy model, testing the potential of the new scheme is presented.

The objectives of this paper are twofold. First, we extend the work presented in E18 and compare the enhanced prior inflation scheme to that of A09, using atmospheric DA experiments. We use the global Community Atmosphere Model (CAM; Neale et al. 2013) in which 6-h forecasts of the atmospheric state, in the troposphere and lower stratosphere, are generated over the month of September in 2010. The second objective is to investigate the applicability, performance and usefulness of adaptive prior and posterior inflation to mitigate filtering issues such as sampling and model errors. In doing so, we attempt to answer the following questions: What inflation scheme is more effective at handling sampling errors? When deficiencies in numerical models dominate bias in ensemble uncertainty estimates, which inflation strategy yields a better fit to the observations? Does inflating both the prior and the posterior ensemble perturbations help mitigate for different error sources? The DA experiments use the latest 'Manhattan' release of the Data Assimilation Research Testbed (DART; Anderson et al. 2009; NCAR/UCAR 2018) and apply the ensemble adjustment Kalman filter (EAKF; Anderson 2003).

Section 2 briefly summarizes the adaptive prior inflation algorithm and then presents the new posterior inflation scheme. To assist the reader, the abbreviations for all inflation methods used in this study are defined in Table 1. Section 3 describes the atmospheric model, the observation sets and the assimilation framework. Results from assimilation experiments, including detailed comparisons of the inflation schemes are also given in section 3. Section 4 provides the summary and conclusions.

\section{Inflation}

Ensemble filtering techniques approximate Bayes's formula using a Gaussian prior, constructed from a set of 
TABLE 1. Definition of all abbreviated inflation methods used in this study.

\begin{tabular}{cl}
\hline \hline Method abbreviation & \multicolumn{1}{c}{ Definition } \\
\hline AI-b & Adaptive prior inflation \\
AI-a & Adaptive posterior inflation \\
mAI-a & Modified adaptive posterior inflation \\
AI-ab & Combined adaptive prior and posterior \\
& inflation \\
CI-a & Constant posterior inflation \\
OPI & Observation-dependent posterior \\
& inflation \\
RTPS & Relaxation to prior spread \\
\hline
\end{tabular}

model realizations, and an associated likelihood distribution. Consider a scalar system in which $x_{b}$ is a random variable describing the background state of the system and denote by $x_{t}$ the true model state. Assume an observation, $y_{o}$, of the state is available:

$$
y_{o}=h\left(x_{t}\right)+\varepsilon_{o}, \quad \varepsilon_{o} \sim N\left(0, \sigma_{o}^{2}\right),
$$

where $h$ is a possibly nonlinear forward operator mapping the true state variable into the observation space and $\varepsilon_{o}$ is an observation Gaussian random error with zero mean and variance $\sigma_{o}^{2}$. The background error $\varepsilon_{b}$ given by: $x_{b}-x_{t}$, is assumed Gaussian centered at zero with variance $\sigma_{b}^{2}$. In statistical linear estimation theory, the analysis (or posterior) expression of the state variable, $x_{a}$, is given by (Jazwinski 1970):

$$
x_{a}=x_{t}+\underbrace{(1-k) \varepsilon_{b}+k \varepsilon_{o}}_{\varepsilon_{a}},
$$

where $\varepsilon_{a} \sim N\left(0, \sigma_{a}^{2}\right)$ denotes the analysis error with variance: $\sigma_{a}^{2}=\sigma_{o}^{2} \sigma_{b}^{2}\left(\sigma_{o}^{2}+\sigma_{b}^{2}\right)^{-1}$, which is also equivalent to $(1-k)^{2} \sigma_{b}^{2}+k^{2} \sigma_{o}^{2}$. The weighting term $k$ is often referred to as the Kalman gain (Kalman 1960). In sequential DA, Eq. (2) is written in terms of the background state and an increment: $x_{a}=x_{b}+\delta x_{b}$ in which $\delta x_{b}$ is a function of $x_{b}, y_{o}$, and $k$.

Within an ensemble framework, the background mean, $\bar{x}_{b}$, and sample variance, $\hat{\sigma}_{b}^{2}$, are as follows:

$$
\begin{aligned}
& \bar{x}_{b}=\frac{1}{N} \sum_{i=1}^{N} x_{b}^{i}, \\
& \hat{\sigma}_{b}^{2}=\frac{1}{N-1} \sum_{i=1}^{N}\left(x_{b}^{i}-\bar{x}_{b}\right)^{2} .
\end{aligned}
$$

Here $N$ refers to the ensemble size and the superscript $i$ denotes the member number. Given the analysis ensemble members after the update, similar expressions can be obtained for the analysis mean, $\bar{x}_{a}$, and sample posterior variance, $\hat{\sigma}_{a}^{2}$. In what follows, we assume that the forecast and analysis ensemble means do coincide with the "true means" obtained by an infinite ensemble. With this assumption, we are only concerned in this study about correcting the bias in the ensemble variances. Detailed information about the inherent bias in the ensemble mean is available in the literature (e.g., Sacher and Bartello 2008; Bocquet 2011). Note here that the sample background and analysis variances are different, usually smaller, than $\sigma_{b}^{2}$ and $\sigma_{a}^{2}$. The goal behind inflation is to bring these sample quantities as close as possible to the theoretical ones. In some scenarios, especially in sparsely observed regions, deflating the ensemble variance as described in E18 is shown to be quite useful. Thus, in what follows the term "variance correction" is used to refer to both inflation and deflation. The forecast (or analysis; $b \mid a)$ ensemble is inflated around the mean as: $x_{b \mid a}^{i}=\sqrt{\lambda} x_{b \mid a}^{i}+(1-\sqrt{\lambda}) \bar{x}_{b \mid a}$ such that $\lambda$ is a scalar variance correction factor.

Given observation $y_{o}$, the forecast (or background) $d_{b}$ and analysis $d_{a}$ innovations are defined:

$$
\begin{aligned}
& d_{b}=y_{o}-h\left(\bar{x}_{b}\right) \approx \varepsilon_{o}-\varepsilon_{b}, \\
& d_{a}=y_{o}-h\left(\bar{x}_{a}\right) \approx \varepsilon_{o}-\varepsilon_{a} .
\end{aligned}
$$

These equations are used in the following sections to derive the likelihoods for the prior and posterior inflation distributions. For simplicity, the derivations are given for a scalar case but can be easily extended for arbitrary dimensions. Further, the observations available for assimilation are processed serially and their observation error distributions are assumed independent (e.g., Bierman 1977).

\section{a. Adaptive prior inflation}

The adaptive prior Inflation (AI-b; "b" refers to background or prior) algorithm is carried out right after the ensemble forecast step. Given an observation, the probability density function (pdf) of the inflation conditioned on the background innovations is computed using Bayes's theorem as follows:

$$
p\left(\lambda \mid d_{b}\right) \propto p(\lambda) p\left(d_{b} \mid \lambda\right) .
$$

The prior density, $p(\lambda)$, is assumed to be normal [i.e., $N\left(\lambda_{b}, \sigma_{\lambda, b}^{2}\right)$ ] following A09 or inverse-gamma \{i.e., $\operatorname{IG}\left[\alpha\left(\lambda_{b}, \sigma_{\lambda, b}^{2}\right), \beta\left(\lambda_{b}, \sigma_{\lambda, b}^{2}\right)\right]$ following E18\}. The mean and variance of the Gaussian pdf are given by $\lambda_{b}$ and $\sigma_{\lambda, b}^{2}$, respectively. The parameters $\alpha$ and $\beta$ define shape and rate of the inverse-gamma prior as a function of the Gaussian first and second moments. The likelihood of the innovations given the inflation, $p\left(d_{b} \mid \lambda\right)$, is assumed normal with the following two moments: 


$$
\begin{aligned}
\mathbb{E}\left(d_{b}\right) & =\mathbb{E}\left(\varepsilon_{o}-\varepsilon_{b}\right)=0, \\
\operatorname{var}\left(d_{b}\right) & =\mathbb{E}\left\{\left[d_{b}-\mathbb{E}\left(d_{b}\right)\right]^{2}\right\}=\sigma_{o}^{2}+\sigma_{b}^{2},
\end{aligned}
$$

assuming that forecast and the observation errors are uncorrelated [i.e., $\mathbb{E}\left(\varepsilon_{o} \varepsilon_{b}\right)=0$ and $\mathbb{E}$ here denotes the expected value]. This may not be true in certain modeling scenarios and thus one could think of decorrelating these quantities before proceeding with the derivation (see, e.g., Hoteit et al. 2015). Next, assume that the ensemble sample variance is related to the optimal one through $\lambda$ as follows: $\sigma_{b}^{2}=\lambda \hat{\sigma}_{b}^{2}$. Substituting this relation in Eq. (9), the likelihood becomes

$$
p\left(d_{b} \mid \lambda\right)=\frac{\exp \left[-\frac{1}{2} d_{b}^{2}\left(\sigma_{o}^{2}+\lambda \hat{\sigma}_{b}^{2}\right)^{-1}\right]}{\sqrt{2 \pi\left(\sigma_{o}^{2}+\lambda \hat{\sigma}_{b}^{2}\right)}} .
$$

As described in A09, the correlation between the inflation factors in space is assumed to be the same as that of the state and the observations. Having constructed both the prior and the likelihood, their product is computed and the updated inflation field can then be generated. More details on this can be found in A09 and E18.

\section{b. Adaptive posterior inflation}

The adaptive posterior Inflation algorithm (AI-a, "a" refers to analysis) is carried out during the update of the state. In light of the previous AI-b derivation, posterior inflation can be computed in a Bayesian framework similar to Eq. (7) according to $p\left(\lambda \mid d_{a}\right) \propto p(\lambda) p\left(d_{a} \mid \lambda\right)$. Note that since the observations are assimilated serially, the analysis ensemble mean and variance change after processing each observation. In AI-b, however, $\bar{x}_{b}$ and $\hat{\sigma}_{b}^{2}$ are obtained during the forecast and remain unchanged while looping over the observations. Below, the mean and variance of the inflation likelihood are evaluated after assimilating the first, second and $j$ th observations. The number-subscript for all variables refers to the index of the observation being assimilated.

- Assimilating the first observation; $y_{o, 1}$ : The analysis innovation is $d_{a, 1}=y_{o, 1}-h_{1}\left(\bar{x}_{a}\right)=\varepsilon_{o, 1}-\varepsilon_{a, 1}$. The moments of $p\left(d_{a, 1} \mid \lambda\right)$ are

$$
\begin{aligned}
\mathbb{E}\left(d_{a, 1}\right) & =\mathbb{E}\left(\varepsilon_{o, 1}\right)-\mathbb{E}\left(\varepsilon_{a, 1}\right)=0 \\
\operatorname{var}\left(d_{a, 1}\right) & =\mathbb{E}\left(\varepsilon_{o, 1}^{2}\right)+\mathbb{E}\left(\varepsilon_{a, 1}^{2}\right)-2 \underbrace{\mathbb{E}\left(\varepsilon_{o, 1} \varepsilon_{a, 1}\right)}_{\neq 0} \\
& =\sigma_{o, 1}^{2}+\sigma_{a, 1}^{2}-2 \mathbb{E}\left[\left(1-k_{1}\right) \varepsilon_{o, 1} \varepsilon_{b}+k_{1} \varepsilon_{o, 1}^{2}\right] \\
& =\left(1-k_{1}\right)^{2}\left(\sigma_{o, 1}^{2}+\sigma_{b}^{2}\right) \\
& =\sigma_{a, 1}^{2} \sigma_{o, 1}^{2} \sigma_{a, 0}^{-2} .
\end{aligned}
$$

Here $k_{1}=\sigma_{b}^{2}\left(\sigma_{b}^{2}+\sigma_{o}^{2}\right)^{-1}$ denotes the Kalman gain after assimilating $y_{o, 1}$, and $\sigma_{a, 0}^{2}$ is equal to $\sigma_{b}^{2}$ and refers to the analysis ensemble variance before assimilating any observation. Likewise, the notation also applies for the errors $\varepsilon_{b}$ and $\varepsilon_{a, 0}$. Imposing $\sigma_{a, 1}^{2}=\lambda \hat{\sigma}_{a, 1}^{2}$, the likelihood function can be written as follows:

$$
p\left(d_{a, 1} \mid \lambda\right)=\frac{\sigma_{a, 0} \exp \left[-\frac{1}{2} d_{a, 1}^{2}\left(\lambda \hat{\sigma}_{a, 1}^{2} \sigma_{o, 1}^{2} \sigma_{a, 0}^{-2}\right)^{-1}\right]}{\hat{\sigma}_{a, 1} \sigma_{o, 1} \sqrt{2 \pi \lambda}} .
$$

- Assimilating the second observation; $y_{o, 2}$ : the innovation at this stage is $d_{a, 2}=y_{o, 2}-h_{2}\left(\bar{x}_{a}\right)=\varepsilon_{o, 2}-\varepsilon_{a, 2}$. The analysis error after assimilating the second observation can be found as $\varepsilon_{a, 2}=\left(1-k_{2}\right) \varepsilon_{a, 1}+k_{2} \varepsilon_{o, 2}$ with zero mean and variance:

$$
\begin{aligned}
\operatorname{var}\left(\varepsilon_{a, 2}\right) \equiv & \mathbb{E}\left(\varepsilon_{a, 2}^{2}\right)=\left(1-k_{2}\right)^{2} \sigma_{a, 1}^{2}+k_{2}^{2} \sigma_{o, 2}^{2} \\
& +2 k_{2}\left(1-k_{2}\right) \mathbb{E}\left(\varepsilon_{a, 1} \varepsilon_{o, 2}\right) .
\end{aligned}
$$

The expectation in the third term on the right-hand side of Eq. (17) vanishes because the observation errors are assumed uncorrelated [i.e., $\mathbb{E}\left(\varepsilon_{o, 1} \varepsilon_{o, 2}\right)=0$ ]. Consequently and given that the expectation of $d_{a, 2}$ is zero, its variance can be deduced as

$$
\begin{aligned}
\operatorname{var}\left(d_{a, 2}\right)= & \mathbb{E}\left(\varepsilon_{o, 2}^{2}\right)+\mathbb{E}\left(\varepsilon_{a, 2}^{2}\right)-2 \mathbb{E}\left(\varepsilon_{o, 2} \varepsilon_{a, 2}\right), \\
\stackrel{\text { Eq. }(17)}{=} & \sigma_{o, 2}^{2}+\left(1-k_{2}\right)^{2} \sigma_{a, 1}^{2}+k_{2}^{2} \sigma_{o, 2}^{2} \\
& -2 \mathbb{E}\left[\left(1-k_{2}\right) \varepsilon_{a, 1} \varepsilon_{o, 2}+k_{2} \varepsilon_{o, 2}^{2}\right], \\
= & \sigma_{a, 2}^{2} \sigma_{o, 2}^{2} \sigma_{a, 1}^{-2} .
\end{aligned}
$$

- Assimilating the $j$ th observation; $y_{o, j}$ : Based on the previous two results, one can easily show that for any observation $j$, the inflation likelihood is proportional to

$$
N\left(0, \lambda \hat{\sigma}_{a, j}^{2} \sigma_{o, j}^{2} \sigma_{a, j-1}^{-2}\right) .
$$

This result indicates that the variance of the inflation likelihood is a function of 1 ) the $j$ th observation error variance, 2) the analysis variance resulting from assimilating $Y_{o, j-1}$ observations, and 3) the current analysis variance given $Y_{o, j}$. The notation $Y_{o, n}=$ $\left[y_{o, 1}, y_{o, 2}, \ldots, y_{o, n}\right]^{\mathrm{T}}$ means all observations up to the $n$th one. Note that this variance equation can be rearranged such that the following form is obtained:

$$
\mathscr{T}(\lambda): \frac{\sigma_{o, j}^{2}}{\mathbb{E}\left(d_{a, j}^{2}\right)}-\frac{\hat{\sigma}_{a, j}^{2}}{\lambda^{-1} \mathbb{E}\left(d_{a, j}^{2}\right)}=1 .
$$

This equation requires that the observation error variance and the sample analysis variance satisfy a 
hyperbola function, $\mathscr{F}$, in which $\lambda$ determines the degree of expansion or contraction of the hyperbola. As $\lambda$ increases, the function further approaches the asymptote: $\hat{\sigma}_{a}=\lambda^{-1 / 2} \sigma_{o}$. Similar to Eq. (9) in E18, in which the prior inflation statistics were found to follow an ellipse instead, this is an interesting result that can be utilized to measure how much the sample statistics are far from the ideal ones.

Using Eq. (21) and a prior distribution for $\lambda$, the adaptive posterior inflation algorithm is thus easy to implement. Essentially, $\lambda$ is updated on the fly each time an observation is assimilated using the product of the prior and the likelihood functions. The update of the moments of the correction factor can be done numerically as described in E18. It is important to note that if the application of the forward operator on the analysis state fails, then the observation cannot be used for updating the inflation. The computational requirements of this algorithm are roughly equivalent to those of AI-b. In terms of storage, the algorithm can be more costly since it requires storing, on top of the mean, the current and the previous analysis variances as shown in Eq. (21). This can become prohibitive in high-dimensional and complex modeling systems. In the following section, a modified and more efficient posterior inflation algorithm is proposed.

\section{c. Modified adaptive posterior inflation, $m A I-a$}

In this algorithm, the state is first updated using the entire set of available observations. The forward operators are then applied to the resulting analysis state to compute the posterior observation estimates:

$$
Y_{a, p}=\left[\begin{array}{c}
h_{1}\left(\bar{x}_{a}\right) \\
h_{2}\left(\bar{x}_{a}\right) \\
\vdots \\
h_{p}\left(\bar{x}_{a}\right)
\end{array}\right] \equiv\left[\begin{array}{c}
y_{a, 1} \\
y_{a, 2} \\
\vdots \\
y_{a, p}
\end{array}\right],
$$

where $p$ is the total number of available observations and $y_{a, j}=h_{j}\left(\bar{x}_{a}\right)$ is the posterior value of the $j$ th observation. Next, loop over all observations:

1) Remove its impact from both the analysis mean and variance as follows:

$$
\begin{aligned}
& \tilde{\sigma}_{a, j}^{2}=\left(\sigma_{a, j}^{-2}-\sigma_{o, j}^{-2}\right)^{-1}, \\
& \tilde{y}_{a, j}=\tilde{\sigma}_{a, j}^{2}\left(y_{a, j} \sigma_{a, j}^{-2}-y_{o, j} \sigma_{o, j}^{-2}\right) .
\end{aligned}
$$

Since the underlying theory indicates that observations at the same time may be assimilated in any order, this is equivalent to assuming that this observation was assimilated last.

2) Calculate the new innovation as: $\tilde{d}_{a, j}=y_{o, j}-\tilde{y}_{a, j}=$ $\varepsilon_{o, j}-\tilde{\varepsilon}_{a, j}$, where $\tilde{\varepsilon}_{a, j}$ is the analysis error after assimilating all observations except for $y_{o, j}$ and can be drawn from $N\left(0, \tilde{\sigma}_{a, j}^{2}\right)$.

3) Construct the Gaussian inflation likelihood function with moments:

$$
\begin{aligned}
\mathbb{E}\left(\tilde{d}_{a, j}\right) & =0 \\
\operatorname{var}\left(\tilde{d}_{a, j}\right) & =\mathbb{E}\left(\varepsilon_{o, j}^{2}\right)+\mathbb{E}\left(\tilde{\varepsilon}_{a, j}^{2}\right)-2 \underbrace{\mathbb{E}\left(\varepsilon_{o, j} \tilde{\varepsilon}_{a, j}\right)}_{=0}=\sigma_{o, j}^{2}+\tilde{\sigma}_{a, j}^{2} .
\end{aligned}
$$

Note that the $j$ th observation error is uncorrelated with the modified analysis error $\tilde{\varepsilon}_{a, j}$ unlike Eq. (12). Compared to Eq. (21), this modified likelihood is only a function of a single analysis variance and is equivalent in form to AI-b's likelihood given in Eq. (9).

4) Update $\lambda$ and its variance using exactly the same procedure as AI-b. Then, repeat all the steps for the next observation.

Compared to AI-a, this algorithm requires an additional evaluation of Eqs. (24) and (25) in the first step; however, its update scheme is equivalent to AI-b. This makes mAI-a's implementation less invasive to available adaptive prior inflation codes. An alternative to this approach would be to withhold some observation set from the assimilation step and use it only to update the inflation but not the state. The presented observationimpact removal strategy might be preferred because it uses all observations to update both the state and the inflation. ${ }^{1}$

\section{d. Lorenz 63 example}

To test the performance of proposed AI-a and mAI-a schemes, we first perform assimilation experiments using the Lorenz 63 system (hereafter L63; Lorenz 1963) with 10 members. The governing equations are as follows:

$$
\begin{aligned}
& \frac{d \xi_{1}}{d t}=\sigma\left(\xi_{2}-\xi_{1}\right), \quad \frac{d \xi_{2}}{d t}=\rho \xi_{1}-\xi_{2}-\xi_{1} \xi_{3}, \\
& \frac{d \xi_{3}}{d t}=\xi_{1} \xi_{2}-\beta \xi_{3},
\end{aligned}
$$

\footnotetext{
${ }^{1}$ This posterior inflation, mAI-a, with the Gaussian prior distribution is what has been implemented in DART since 2009 but it was never documented in a rigorous mathematical framework.
} 
TABLE 2. EAKF assimilation results for different posterior inflation techniques obtained using the L63 system with 10 members. Observations of variables $\xi_{1}, \xi_{2}$, and $\xi_{3}$ are available every 10 time steps with an observation error variance of 1 . The reported metrics are mean squared error of the forecast (MSE-b) and analysis (MSE-a). VAR stands for the variance of the ensemble. The consistency of the ensemble estimates is computed as the ratio of MSE and VAR. Inflation values are temporal averages over 10000 steps assimilation run.

\begin{tabular}{lccccc}
\hline \hline & CI-a & AI-a & mAI-a & OPI & RTPS \\
\hline MSE-b & 0.1715 & 0.1659 & 0.1668 & 0.1654 & 0.1748 \\
MSE-a & 0.0780 & 0.0740 & 0.0758 & 0.0756 & 0.0784 \\
MSE-b/VAR-b & 0.8241 & 1.0511 & 1.0117 & 0.9363 & 0.9949 \\
MSE-a/VAR-a & 0.9209 & 1.0199 & 0.9759 & 0.9235 & 0.9780 \\
Inflation & 1.033 & 1.0077 & 1.0139 & 1.0185 & 1.0121 \\
\hline
\end{tabular}

where $\sigma=10, \rho=28, \beta=2.67$, and $d t=0.01$ is the model time step.

\section{1) Perfect model}

We assume perfect modeling conditions and assimilate observations of all variables $\left(\xi_{1}, \xi_{2}\right.$, and $\left.\xi_{3}\right)$ every 10 time steps for a total of 10000 assimilation cycles. In addition to the AI-a and mAI-a schemes, we perform three more experiments: 1 ) using constant posterior inflation (CI-a), 2) using the observation-dependent posterior inflation (OPI; Hodyss et al. 2016), and 3) the RTPS scheme of Whitaker and Hamill (2012). The goal is to validate the performance of the proposed posterior inflation schemes using results from methods that have been tested extensively in earlier literature studies. For each experiment, the parameters of the different schemes are tuned for best performance. For instance, the optimal parameters required by the OPI scheme, using 10 members, were found as $a=0.9$ and $b=2$ (consistent with the recommendation of the authors of the scheme; i.e., $a<1$ and $b>1$ ). The proposed adaptive schemes use the enhancements presented in E18. The resulting time-averaged mean squared errors of the background (MSE-b) and the analysis (MSE-a) estimates are given in Table 2. Also tabulated are the ensemble consistency values: [MSE/VAR (ensemble variance)] and the overall posterior inflation averages:

$$
\begin{aligned}
\mathrm{MSE} & =\frac{1}{3 L} \sum_{\ell=1}^{L} \sum_{r=1}^{3}\left[\overline{\mathbf{x}}_{e}(r, \ell)-\mathbf{x}_{t}(r, \ell)\right]^{2}, \\
\mathrm{VAR} & =\frac{1}{3 L(N-1)} \sum_{\ell=1}^{L} \sum_{r=1}^{3} \sum_{i=1}^{N}\left[\mathbf{x}_{e}^{i}(r, \ell)-\overline{\mathbf{x}}_{e}(r, \ell)\right]^{2} .
\end{aligned}
$$

The subscript $e$ denotes an ensemble estimate which can be either forecast or analysis. $r$ represents an element in the state, $\mathbf{x}$ (i.e., $\xi_{1}, \xi_{2}$, or $\xi_{3}$ ), $\ell$ denotes time, and $L$ is total number of assimilation cycles. For a consistent
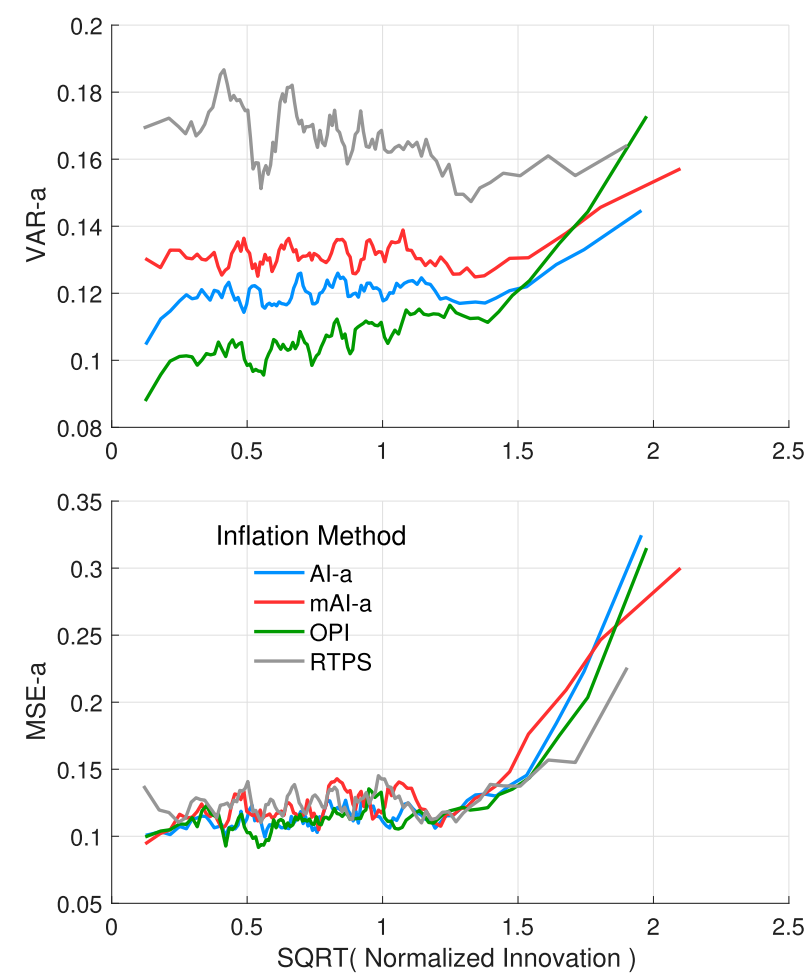

FIG. 1. (top) The analysis ensemble variance VAR-a and (bottom) mean squared errors MSE-a as a function of the square root of the normalized innovations. The curves are results from four L63 assimilation runs using AI-a, mAI-a, OPI, and RTPS inflation schemes.

ensemble statistics, we expect the consistency to be close to 1 . The results from the proposed adaptive posterior inflation schemes are more accurate than those obtained using constant inflation. Assessing the consistency values, the background variance suggested by CI-a, using $\sqrt{\lambda}=1.033$, is underestimated. AI-a performance is found to be only marginally better than mAI-a and both schemes yield consistent forecast and analysis estimates. On average, the schemes suggest around 1.01 posterior inflation. Furthermore, the performance suggested by AI-a and mAI-a is comparable to that of OPI. The proposed schemes outperform RTPS in terms of the accuracy of the estimates; however, RTPS is shown to yield the best consistency values.

To get more insights on the behavior of the algorithms, we bin in Fig. 1 both MSE-a and VAR-a as function of the normalized innovations as in Hodyss et al. (2016). It is seen that the posterior ensemble variance proposed by AI-a, mAI-a and OPI is function of innovation and is generally correctly predicting the MSE-a. When the innovations are large the posterior variance is also large, and when the innovations are small the posterior variance is relatively small. The response to large innovations, suggested by AI-a and 

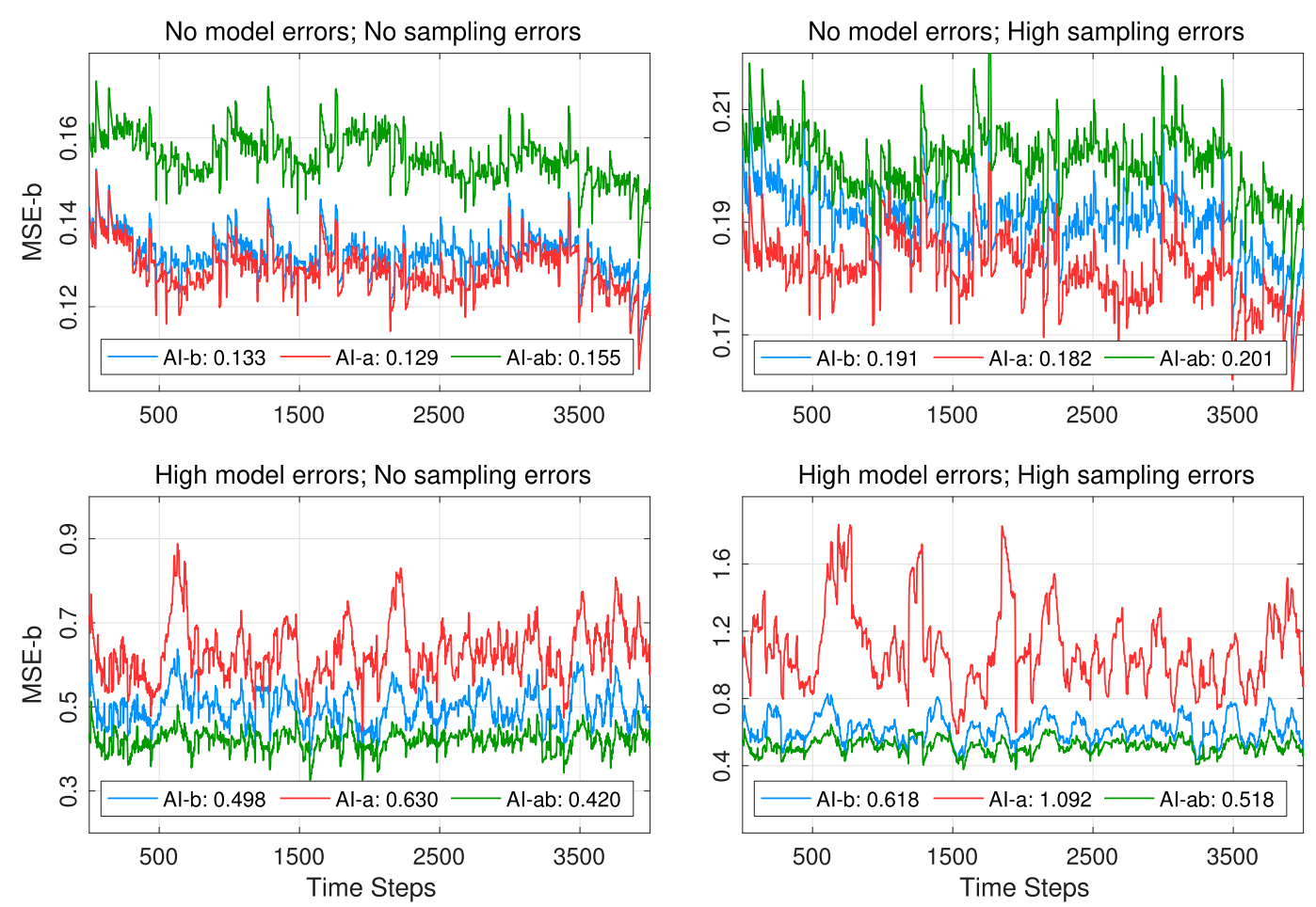

FIG. 2. Moving averages (over 100 model steps) of background mean squared errors, MSE-b, resulting from three assimilation experiments in the L63 model: EAKF equipped with 1) prior inflation, AI-b, 2) posterior inflation, AI-a, and 3) both prior and posterior inflation, AI-ab. The error curves are displayed over the last 4000 assimilation cycles only. Sampling errors increase from left panels to the right and model errors increase from top panels to the bottom.

mAI-a, is not as strong as that of OPI because of the prior inflation information given by the Bayesian update. The variance resulting from RTPS, on the other hand, is not a function of the innovations and this is clearly shown on the plot. The MSE resulting from all schemes is a strong function of the innovations.

\section{2) IMPERFECT CONDITIONS}

The performance of the mAI-a (hereafter AI-a) is validated under imperfect assumptions by simulating both sampling and model errors. Four different sampling and model error scenarios are conducted (Fig. 2). To investigate the effect of sampling errors, the assimilation is first run with 5 members and later with 5000 members. To add model error, the three parameters (i.e., $\sigma, \rho$, and $\beta)$ are perturbed with a Gaussian noise of zero mean and 0.5 standard deviation. The performance of AI-a is compared to: (Fig. 2a) AI-b and (Fig. 2b) both AI-b and AI-a combined, denoted as AI-ab. Under perfect modeling conditions and near-zero sampling errors (i.e., $N=5000$ ), both prior and posterior inflation are able to produce high-quality estimates. Combining both adaptive inflation schemes slightly degrades the MSE of the estimates. The reason for that may be related to the overinflated ensemble variance. We measured the average consistency from the $\mathrm{AI}-\mathrm{ab}$ run and found it to be 1.61. It seems that for an error-free system, injecting extra uncertainty in the ensemble is unnecessary and should be avoided. However, this is never the case in realistic scenarios. When sampling errors increase (i.e., $N=5$ ), the importance of using AI-a is strongly highlighted. On average, AI-a estimates are $5 \%$ and $10 \%$ more accurate than those obtained using AI-b and AI-ab, respectively. Introducing model errors to the filter strongly degrades the performance of AI-a. AI-b, on the other hand, suggests better handling of model errors outperforming AI-a both in the presence and absence of sampling errors. Interestingly, AI-ab yields the best performance in presence of model errors suggesting around $15 \%$ improvement in accuracy over AI-b estimates. This indicates that AI-a algorithm may not be well-suited for systems with high model errors; however, combining it with prior inflation can still help mitigate for sampling errors and thus improve the quality of the prediction. In terms of computational complexity, we found that AI-a is only marginally more expensive to run compared to AI-b. The extra wall-clock time required to perform a full DA cycle using AI-a is around $3 \%$ of the total computing time required by AI-b. 


\section{Atmospheric GCM assimilation}

\section{a. The model}

The model we use in this study is the Community Atmosphere Model, version 4 (CAM4), released as the atmospheric component of the Community Climate System Model (CCSM4, Gent et al. 2011). CAM4 uses the finite volume (FV) dynamical core (Lin and Rood $1996)$ with a horizontal resolution of $1.9^{\circ} \times 2.5^{\circ}$. It has 26 hybrid sigma and pressure levels, which are terrain following at the surface, with a transition to pure pressure at $\sim 85 \mathrm{hPa}$. The top of the model is at approximately $2 \mathrm{hPa}$. The default horizontal diffusion has been replaced by a fourth-order divergence damping operator throughout the model depth (CAM namelist option div24del2flag $=4$ ). The goal is to improve the performance of this climate model when it is used for short forecasts from initial conditions adjusted by data assimilation (Lauritzen et al. 2012). The lack of extra damping near the model top led us to neglect observations above $50 \mathrm{hPa}$ in order to avoid assimilation where the model is known to be deficient.

The major physical parameterizations include: 1) Zhang and McFarlane deep convection, modified (from CAM3.5) to include a dilute plume calculation and Convective Momentum Transport; 2) Hack shallow convection; 3) prognostic condensate; 4) prognostic precipitation; 5) shortwave and longwave radiation "CAMRT"; and 6) prescribed, radiatively active aerosols and greenhouse gases. Ancillary models include a data ocean model using monthly mean SSTs interpolated in time to the model date (Hurrell et al. 2008), prescribed sea ice concentrations, a thermodynamic sea ice model, and a fully active Community Land Model (Oleson et al. 2008; Dickinson et al. 2006). Time integration of the model is Eulerian and the step is $10 \mathrm{~min}$.

The state variables in the assimilation framework are surface pressure (PS), sensible temperature $(T)$, wind components $(U$ and $V$ ) on staggered grids (D grid, Arakawa and Lamb 1981), specific humidity $(Q)$, cloud liquid water (CLDLIQ), and cloud ice (CLDICE).

\section{b. Observations}

Observations are assimilated at 0000, 0600, 1200, and 1800 UTC on a daily basis. The assimilated observations include GPS radio occultation refractivity data (GPSRO; Satellite Data Analysis and Archive Center 2018) and NCEP-GDAS (Global Data Assimilation System) products such as temperature and winds from radiosondes, aircraft, and ACARS, in addition to satellite cloud motion wind vectors (NCAR/UCAR 1994). Except for GPS-RO observations, all other assimilated observations were used for the NCEP-NCAR reanalysis

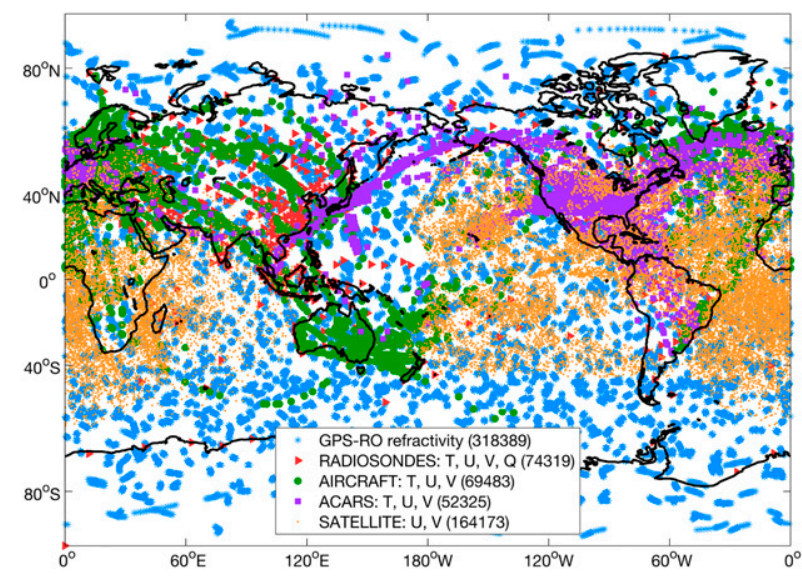

FIG. 3. Sample observation network used for assimilation in DART. The network is valid at 0000 UTC 6 Sep 2010. The total number of observations is reported for each kind in the legend. For visual purposes, the density of the observations has been refined by a factor of 7 .

project (Kistler et al. 2001). Radiosonde moisture observations are not assimilated and only evaluated for diagnostic purposes. No satellite radiances are assimilated in this study. A sample observing network valid at 0000 UTC 6 September 2010 is plotted in Fig. 3. All observations available within a window of $\pm 3 \mathrm{~h}$ around each analysis time are assimilated.

Observation errors are assumed independent for all observations. Observation error standard deviations for conventional $T, U$, and $V$ data are specified as in the NCEP-GDAS. For refractivity data, the observation error variances are the same as those used in the NCEP Gridpoint Statistical Interpolation analysis system (GSI, Cucurull 2010). In time, the largest number of observations is available at 0000 and 1200 UTC.

\section{c. Experimental design and implementation}

CAM4 is first initialized at 0000 UTC 1 January 2010 using a model output file that is part of the CAM package. The model is then propagated freely for 8 months until the first of August 2010. A total of 80 independent random draws from a Gaussian distribution with $0.1-\mathrm{K}$ standard deviation are added to each temperature grid point. Data assimilation cycles from 0600 UTC 1 August to1800 UTC 30 September 2010; however, we only report assimilation results from the month of September to avoid any transient behavior. In Fig. 4, the ensemble standard deviation at 0000 UTC 15 August 2010 for PS and $V$ is shown. High PS ensemble spread $(\sim 10 \mathrm{hPa})$ is present in the Southern Hemisphere while smaller variations are seen in the tropical zones. As for $V$, the variability in the ensemble is largest in the Northern Hemisphere. In the tropics and 

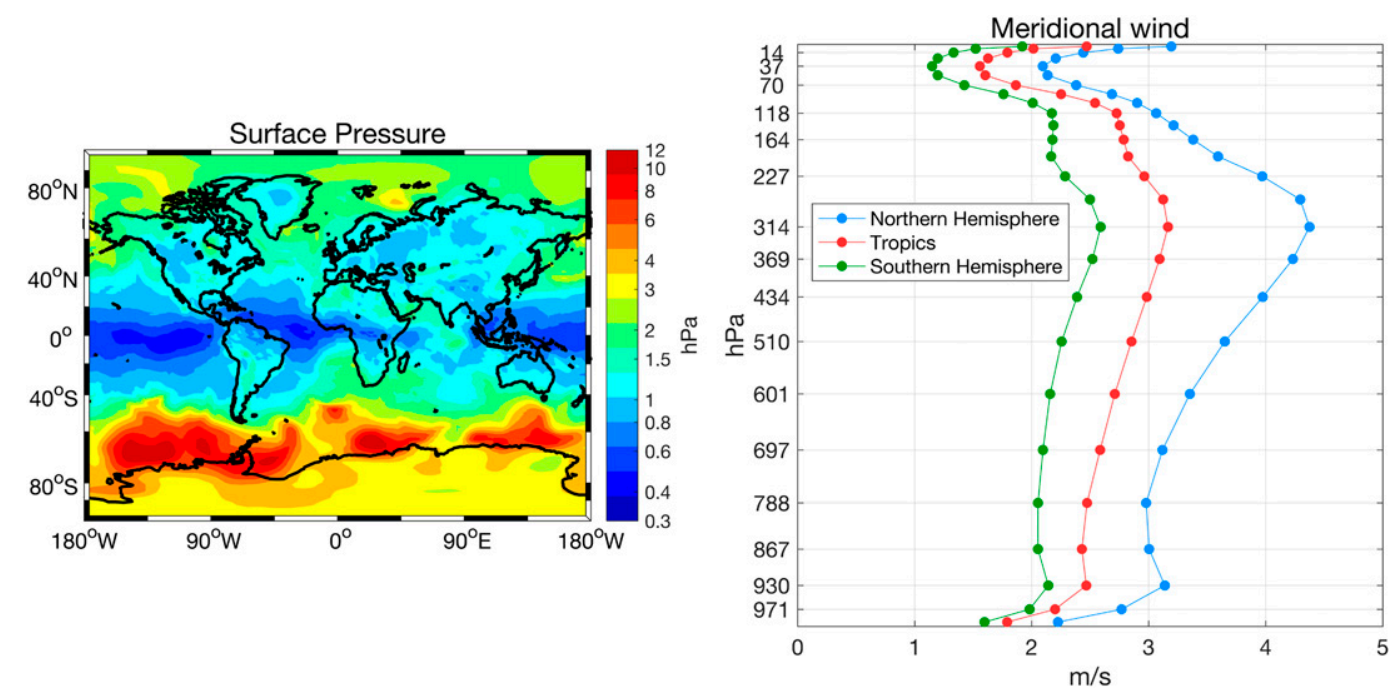

FIG. 4. Ensemble standard deviation of (left) surface pressure and (right) meridional velocity valid at 0000 UTC 15 Aug 2010. The color bar is given in log scale. The markers in the right panel denote a spatial average of the ensemble spread obtained at all points in the Northern Hemisphere $\left(20^{\circ}-90^{\circ} \mathrm{N}\right)$, tropics $\left(20^{\circ} \mathrm{S}-20^{\circ} \mathrm{N}\right)$, and Southern Hemisphere $\left(90^{\circ}-20^{\circ} \mathrm{S}\right)$.

the Southern Hemisphere, the ensemble spread is about $2.5 \mathrm{~m} \mathrm{~s}^{-1}$. Along height, the variability increases going up in the troposphere before it starts decreasing again in the stratosphere.

While cycling, the land component of CESM (CLM) is not directly affected by the assimilation; however, it is forced by the updated atmosphere. The observation quality control flags provided by NCEP, in addition to those within DART, are used. If, for example, an observation is not considered in the NCEP-NCAR reanalysis project then it is not assimilated in DART. The quality control in DART compares observations with the prior ensemble mean and ignores observations with innovations larger than 3 times the total spread (i.e., $\sqrt{\sigma_{o}^{2}+\sigma_{b}^{2}}$ ). It also excludes observations below the lowest model level and above $50 \mathrm{hPa}$. The latest "Manhattan" version of DART (subversion revision number 12758), released on 17 March 2017, is used in this study.

The impact of each observation is localized based on the distance between the observation and the state variables. We use a fifth-order polynomial function of Gaspari and Cohn (1999) with a horizontal half-width of $0.15 \mathrm{rad}(\approx 960 \mathrm{~km})$. In the vertical, the localization is done using scale height coordinates. The scale height is defined to be $\log _{10}\left(P_{0} / p\right)$, so that it is 0.0 at pressure $P_{0}$ $\left(10^{5} \mathrm{~Pa}, \sim\right.$ sea level) and increases with height (as $p$ decreases). For instance, the top of the model is located at 2.69 scale heights. In our experiments, the half-width of the Gaspari Cohn profile is 0.375 scale heights. Concerning inflation, the prior densities required by the adaptive prior and posterior schemes are initialized with mean $\lambda=1$ and standard deviation $\sigma_{\lambda}=0.6$ and the inflation standard deviation is kept constant throughout the entire assimilation period.

\section{d. Prior inflation}

Spatially and temporally varying adaptive prior inflation of A09 and E18 are compared in this section. The comparison is made using the common observations that were assimilated by both schemes. Plotted in Fig. 5 is a summary of the background root-mean-square error (RMSE) difference between two assimilation experiments, one using A09's scheme and the other using E18's. The RMSE difference is calculated over 3 regions (Northern Hemisphere, tropics, and Southern Hemisphere) and along 14 vertical level bins extending from the surface to the top of the model. For $U$ and $V$, E18 consistently outperforms A09 minimizing the bias and pushing the prediction closer to the observations. This is demonstrated over the entire vertical domain for radiosondes and around the tropopause for aircraft, ACARS, and satellite winds. As for temperature, the largest improvements suggested by E18 are for radiosondes in the Southern Hemisphere toward the top of model and for near-surface AIRCRAFT especially in the tropics. For ACARS $T$, the performance of both schemes seems to match. Assessing refractivity estimates, there is a clear advantage for E18 in the boundary layer of tropical areas. Overall, E18 is shown to yield higher-quality estimates for all assimilated observation kinds. 

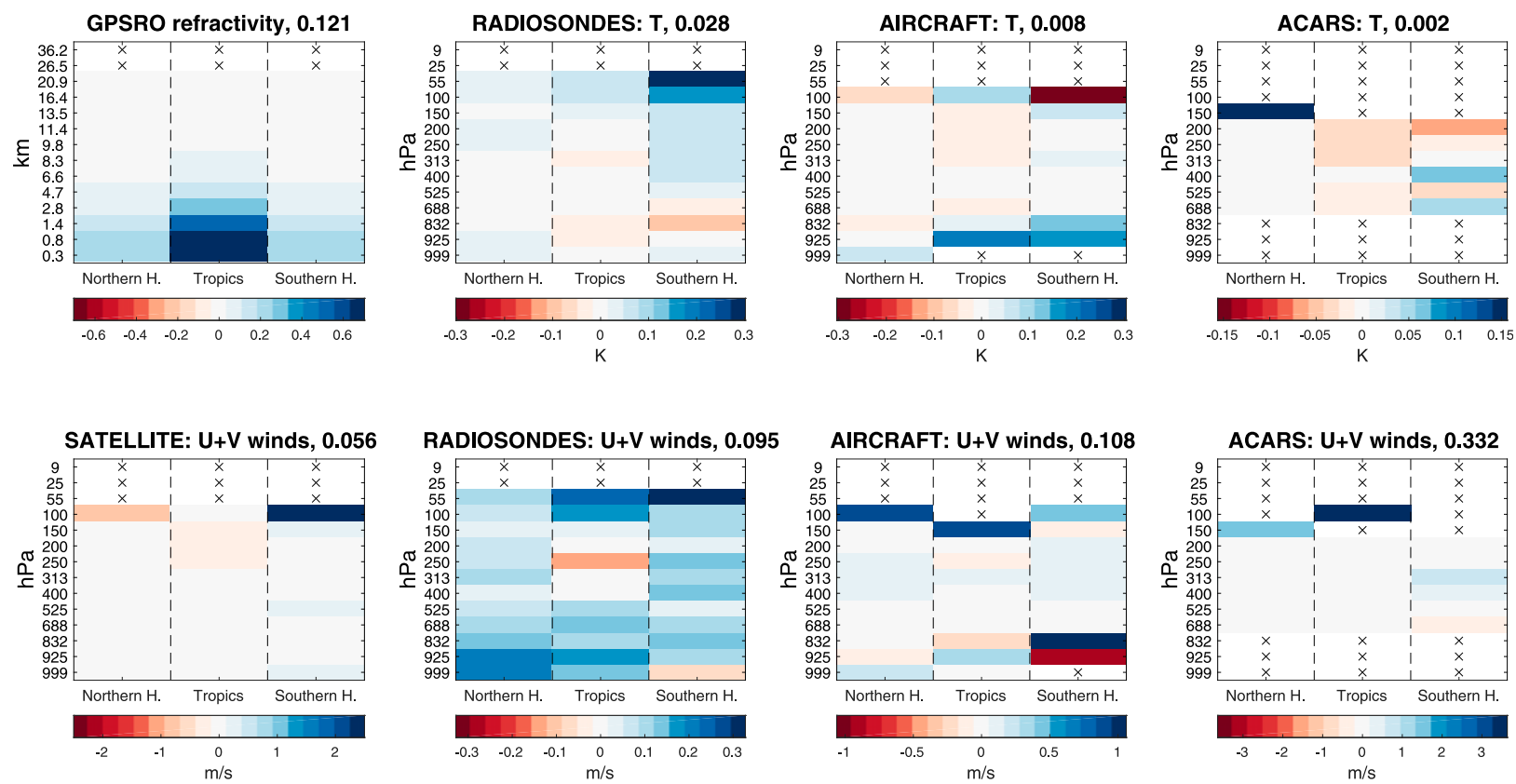

FIG. 5. Spatially and temporally averaged RMSE difference between E18 and A09 for refractivity, temperature, and winds. The horizontal axis denotes the geographic regions: Southern Hemisphere, tropics, and Northern Hemisphere. The vertical axis shows the different level bins ( $\mathrm{hPa}$ or $\mathrm{km}$ ) along which the RMSE values are computed. The overall mean of the weighted RMSE difference (weighting is based on the number of observations in each bin to the total number of assimilated observations) is shown on top of the panels. Blue (red) color means RMSE of A09 is bigger (smaller) than that of E18. A positive overall weighted mean indicates that E18 is more accurate and vice versa. The symbol $(\times)$ on the plots means there are no observations.

In Fig. 6 (top panel), the consistency of radiosonde surface $T$ in the Northern Hemisphere is displayed. With no inflation, the background ensemble spread is smaller than the prediction errors yielding a consistency of 0.75 . Adding prior inflation significantly improves the ensemble estimates resulting in 1.17 and 1.09 averaged consistencies for A09 and E18, respectively. Compared to A09, E18 produces a slightly better match between the ensemble spread and the RMSE. The consistency obtained using E18 for all other variables (not reported) is found close to 1 . Surface pressure inflation maps resulting from both prior inflation schemes are compared in Fig. 6 (bottom panel). Inflation values suggested by A09 in the Northern Hemisphere (North America and Europe) and the tropics are larger than those of E18. This is also observed in various parts of the Indian Ocean and Australia. The observation network in these regions is dense compared to other parts of the world. In less densely observed areas such as the Arctic Ocean and Antarctica, the performance of both inflation schemes is essentially the same and thus, the inflation patterns are similar.

The enhancements to the prior and likelihood distributions proposed in E18 are shown to be useful. One important feature of E18 is the use of an inverse-gamma distribution, which allows for slight deflation and prevents the occurrence of unphysical (negative) values of inflation.
This reduces excessive inflation in densely observed regions while maintaining small prediction errors. In sparse network areas where the uncertainty often grows due to the lack of observations (e.g., Southern Ocean), E18 suggested modest deflation thus shrinking the large uncertainty in the ensemble. In summary, E18 generated satisfactory CAM predictions and consistently outperformed A09 for the tested assimilation period. This coincides with the discussion and the conclusions drawn from the OSSE experiments in the previous study (i.e., E18). Accordingly, we adopt E18 enhancements for all adaptive inflation results described in the following sections.

\section{e. Posterior inflation}

As an extension to the findings in the L63 model, the proposed adaptive posterior inflation (i.e., AI-a) is compared here to RTPS. The scaling factor of the RTPS scheme has been tuned for best performance $(\alpha=1)$. Small values of the scaling factor produced low-quality estimates. For values larger than 1, we experienced instabilities calculating energy fluxes in CAM4. Overall, the proposed AI-a was able to assimilate at least $10 \%$ more observations than RTPS. Figure 7 plots a summary of background RMSE difference between AI-a and RTPS, using their common observations, similar to Fig. 5. It is seen that ACARS, aircraft, and satellite 

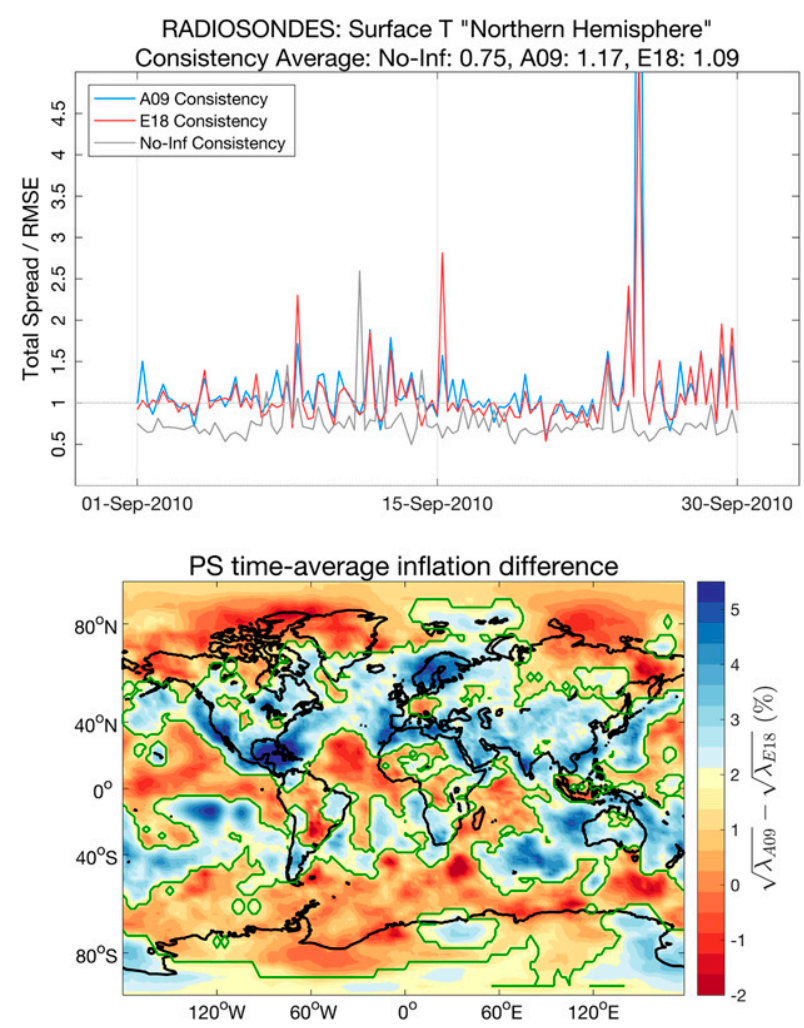

FIG. 6. (top) Consistency (spread divided by RMSE) of surface radiosonde $T$ estimates in the Northern Hemisphere resulting from A09, E18, and a run that does not use inflation. (bottom) Average, over the month of September, PS inflation map difference between A09 and E18. The green contours correspond to locations where $\sqrt{\lambda_{\mathrm{A} 09}}$ is larger than $\sqrt{\lambda_{\mathrm{E} 18}}$ by more than $2 \%$.

temperature and wind estimates resulting from AI-a are more accurate than those of RTPS. Except for refractivity, AI-a estimates are systematically better in the Northern Hemisphere and the tropics. RTPS only outperforms AI-a in the upper layers of the Southern Hemisphere as can be shown for radiosonde $T$ and $U+V$. Furthermore, AI-a retained slightly larger posterior variance yielding flatter rank histograms for most of the diagnosed atmospheric variables (not shown).

It is possible to obtain improved results using RTPS if $\alpha$ was to change adaptively in time (e.g., Ying and Zhang 2015). This was not tested in the current study and thus the presented comparison should not be utilized as a proof that AI-a is a better method than RTPS. In the following section, $\mathrm{AI}-\mathrm{a}$ is compared to AI-b in the context of bias mitigation.

\section{f. Model bias treatment}

Running a CAM4 filtering system with no special treatment for bias may yield catastrophic results such as filter divergence. In Fig. 8 (left panel), the line plot denotes the time-average absolute bias in different atmospheric variables, relative to the various observation types. This is obtained by running a free CAM4 ensemble with no data assimilation. The terms $U$ and $V$ consist of the largest biases (relative to $T$ ) especially those predicted at aircraft, ACARS, and satellite locations. In the case of no inflation, the ensemble variance is strongly underestimated causing huge discrepancies between the ensemble estimate and the observations. Two rank histogram plots for radiosonde and ACARS winds in the tropics at $250 \mathrm{hPa}$ are shown in Fig. 8 (right panels). The rank histograms are U-shaped meaning that most of the observations fall outside the span of the ensemble and thus the spread is too small. As a result, the assimilation algorithm fails to assimilate large number of observations (as shown in the left panel) resulting in an unsuccessful filtering exercise. The use of inflation strongly enhances the performance of the filter. The total number of assimilated GPS-RO refractivity observations is around 5 million, which is more than twice the number used in the no-inflation run. As for the wind rank histograms, both AI-b and AI-ab successfully recover an almost flat-shaped histogram. AI-a suggests a clear improvement to the histogram shape; however, the ensemble is still not fully able to accommodate as many observations as the other inflation schemes.

Figure 9 plots the bias profiles of the prior and posterior specific humidity (i.e., $Q$ ) estimates resulting from No-Inf, AI-b, AI-a, and AI-ab runs. Recall that radiosonde $Q$ observations are not assimilated so they are useful for comparing the potential of the inflation schemes to mitigate for model bias because they are less susceptible to overfitting the data. As can be seen, there is a positive moisture bias in CAM4 near the boundary layer. In a densely observed Northern Hemisphere, AI-b outperforms AI-a and reduces the prior and posterior biases by about $50 \%$. The number of assimilated observations in the tropics is smaller and the bias in the model is the highest, growing up to $\sim 0.8 \mathrm{~g} \mathrm{~kg}^{-1}$ near the surface. AI-a is found more effective than AI-b in this region. The overall weighted prior bias resulting from AI-b and AI-a is 0.06 and $0.03 \mathrm{~g} \mathrm{~kg}^{-1}$, respectively. In the Southern Hemisphere, AI-a performs poorly in contrast to prior inflation which removes large portions of the bias in lower troposphere areas. In the upper levels $(\leq 400 \mathrm{hPa})$ of the model, AI-a is seen to yield smaller biases than AI-b. The reason for this is unclear though it might be related to the model damping described earlier. In all regions, the best performance is suggested by AI-ab (and AI-b in the Southern Hemisphere) roughly canceling the entirety of the bias as demonstrated in the Northern Hemisphere. Investigating the variance of the prior and posterior ensembles reveals that AI-ab is able to retain the biggest spread. These results suggest that in such a biased system, inflating both prior and the posterior ensemble is 

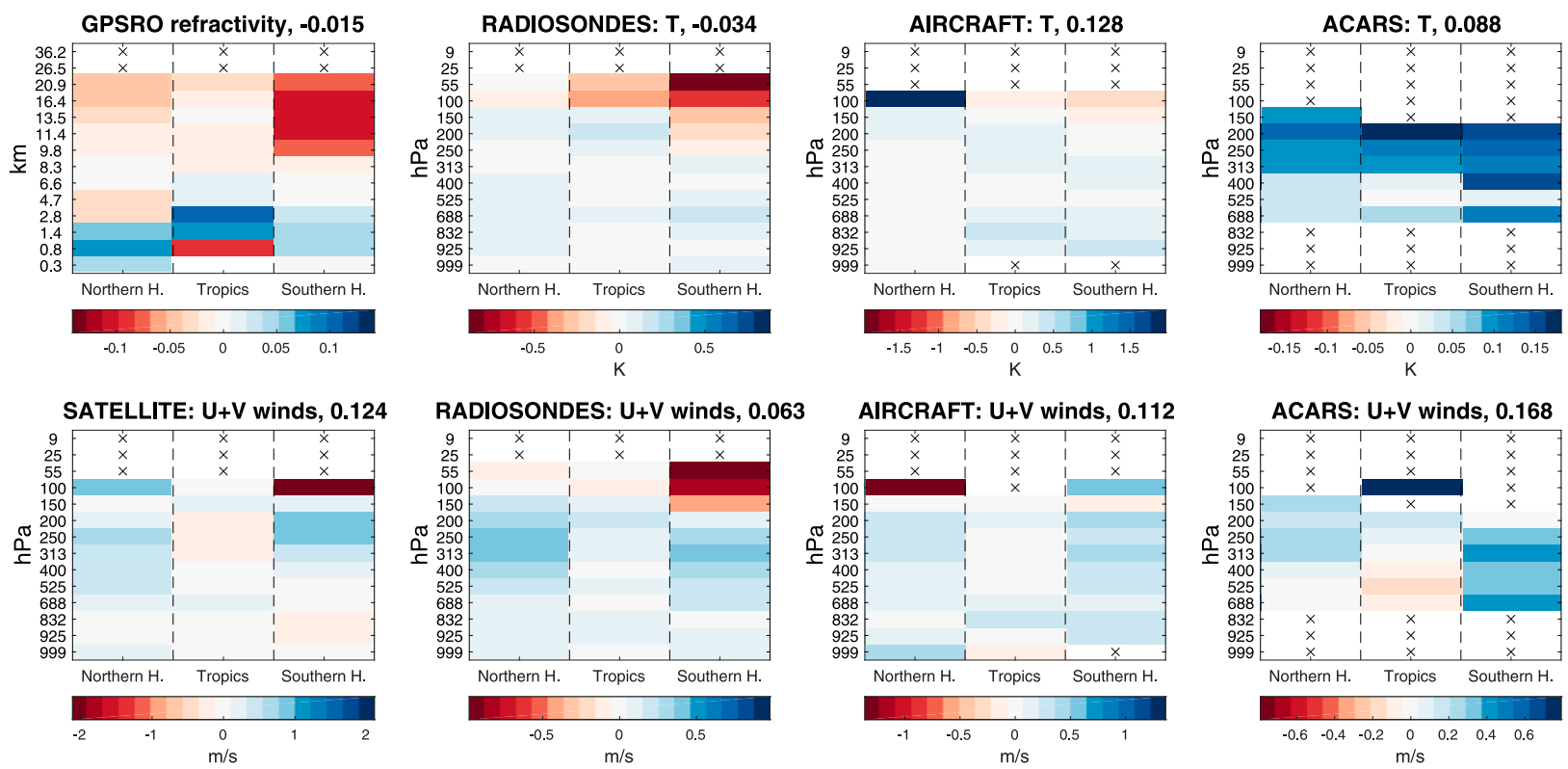

FIG. 7. As in Fig. 5, but comparing AI-a and RTPS. Blue (red) color means RMSE of RTPS is bigger (smaller) than that of AI-a. A positive overall weighted-mean indicates that $\mathrm{AI}-\mathrm{a}$ is more accurate and vice versa.

useful and can help bring the model's prediction and the filter's analysis closer to the observations.

Figure 10 compares the absolute bias obtained using AI-b to that of AI-ab for all assimilated observations. It is shown that AI-ab is able to produce better estimates especially for high biased variables such as AIRCRAFT and satellite winds (refer to the left panel of Fig. 8). Comparable performance is observed for less biased variables such as GPS-RO refractivity. Consistent with the patterns in Fig. 7, radiosonde estimates of AI-ab in the upper layers of the Southern Hemisphere are less accurate than AI-b ones. This may indicate that overinflating sparsely observed regions can degrade the accuracy of the prediction. In general, combining prior and posterior inflation is shown to decrease the bias and enhance the performance of the filter for most of the variables.

\section{Discussion}

The difference in the performance of AI-b, AI-a, and AI-ab can be attributed to many factors. One of which is the information content (IC), $\partial \bar{x}_{a} / \partial y=\left(\sigma_{o}^{2}+\sigma_{b}^{2}\right)^{-1} \sigma_{b}^{2}$ (Cardinali et al. 2004). The IC roughly determines how much weight is placed on the observations during the analysis given the ensemble's prior variance and the uncertainty in the observations. Thus with low-quality background estimates, more weight on the observations is generally desirable to obtain good analysis. In our experimental setup, AI-b is shown to place more emphasis on the data compared to AI-a (Fig. 11). For instance, the IC for refractivity suggested by AI-b is around 0.7 (i.e., $70 \%$ of the update information is retrieved from the data while the rest comes from the background) and only 0.3 by AI-a. The reason for this behavior could be related to the fact that background innovations are usually larger than the posterior ones. Since AI-a uses the posterior innovation to update the inflation, the size of the Kalman correction it yields is generally smaller than the prior inflation case. Compared to AI-b and AI-a, AI-ab has the largest IC allowing the algorithm to extract more information from the data. We note that this feature may not be attractive for observations with large uncertainties.

\section{Spatial patterns and inflation structure}

The spatial patterns of the inflation maps, the state increments and ensemble spread are studied in this section. Figure 12 maps the average $T$ increments for a model level approximately at $697 \mathrm{hPa}$ obtained during the last two weeks in September 2010 using AI-b (Fig. 12a), AI-a (Fig. 12c) and AI-ab (Fig. 12e). The $T$ average ensemble spread during the same period is shown in the second column of Fig. 12. In Fig. 13, the average inflation maps (Figs. 13a,d,e,f) and the time correlation between AI-ab inflation and those of AI-b (Fig. 13b) and AI-a (Fig. 13c) are shown. The major updates for $T$ at this elevation happen in the southern and northern extratropics. These persistent changes in $T$ may not be the largest. Other locations probably have larger increments of both signs that cancel each other when averaged over time. In the AI-b run, areas such as South Africa and the Indian Ocean are strongly inflated 

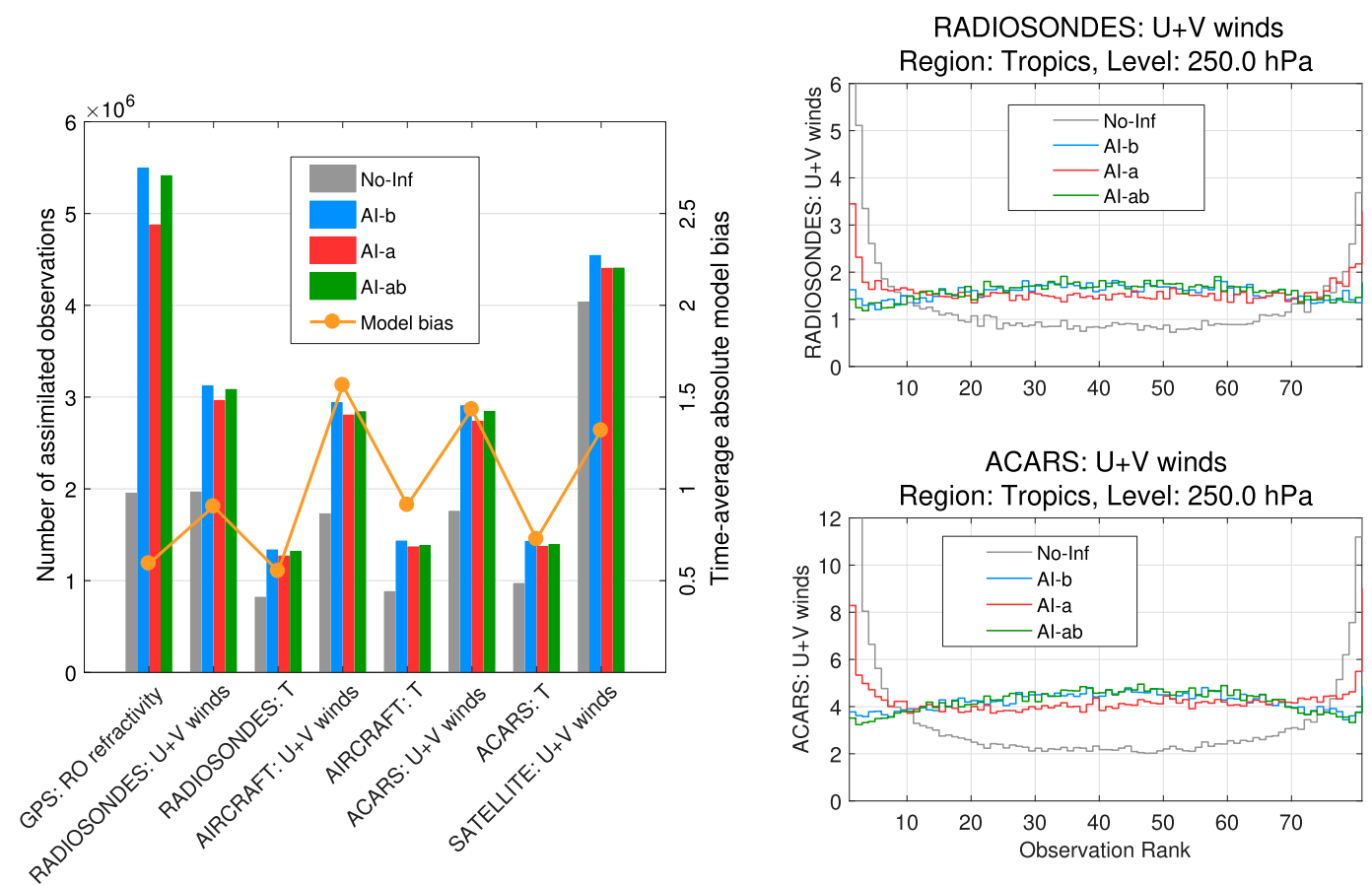

FIG. 8. (left) The total number of assimilated observations in September 2010 resulting from four assimilation runs (left $y$ axis): 1) with no inflation, 2) using AI-b, 3) using AI-a, and 4) using AI-ab. According to the right $y$ axis, the line depicts the absolute bias (relative to the average bias from all observations) for RO refractivity, $T, U$, and $V$ data. (right) Rank histograms (top view) plots for radiosonde and ACARS winds at elevation $250 \mathrm{hPa}$ in the tropics resulting from the four assimilation runs. The rank histogram data are averaged over the month of September.

resulting in large ensemble spread and numerous large positive and negative temperature increments. AI-a, on the other hand, suggests increasing the ensemble variance over East Asia through inflating the analysis ensemble by a factor of 2. Prior and posterior temperature ensembles around the Arctic and the Antarctic Circles experience a $20 \%$ deflation which could be attributed to the sparsity of observations. This is reflected by a strong cooling pattern at very low latitudes, given CAM4's warming bias. Overall, the sign and pattern of the $T$ increments suggested by AI-a and AI-b are similar; however, their magnitude is quite different. As shown, AI-b imposes bigger local updates, yet on average the global change in $T$ in both runs is comparable (i.e., $<0.5 \mathrm{~K}$ ); $T$ increments suggested by AI-ab follow similar patterns as those obtained using prior/posterior inflation only, and $T$ ensemble spread at $697 \mathrm{hPa}$ resulting from AI-a is the smallest especially in the Caribbean region.

We observe that $\sqrt{\lambda_{a b}^{b}}$ (prior inflation of $\mathrm{AI}-\mathrm{ab}$ ) and $\sqrt{\lambda_{a b}^{a}}$ (posterior inflation of AI-ab) are highly correlated with $\sqrt{\lambda_{b}}$ (prior inflation of AI-b) and $\sqrt{\lambda_{a}}$ (posterior inflation of AI-a), respectively. For instance, at $800 \mathrm{hPa}$ the correlation between $\sqrt{\lambda_{a}}$ and $\sqrt{\lambda_{a b}^{a}}$ is approximately 0.9 . This correlation suddenly drops between 600 and $100 \mathrm{hPa}$ to $\sim 0.5$ and then it is shown to increase again in the stratosphere. Correlation between $\sqrt{\lambda_{b}}$ and $\sqrt{\lambda_{a b}^{a}}$ behaves almost exactly in an opposite manner. Correlation decreases to 0.7 at $700 \mathrm{hPa}$ and then gradually increases reaching 0.85 at $200 \mathrm{hPa}$ before it starts decreasing again near the tropopause.

Averaging in space and time, the magnitude of $\sqrt{\lambda_{b}}$ is found larger than $\sqrt{\lambda_{a}}$. Although both AI-b and AI-a algorithms are initialized using the same parameters and distribution, background ensemble perturbations seem to require more inflation than does the analysis ensemble. This may be related to the fact that after the update, the analysis mean often gets closer to the observations thus reducing the discrepancy between the likelihood and prior inflation pdfs. In contrast, the forecast innovations are usually bigger. Compared to AI-ab inflation fields, $\sqrt{\lambda_{b}}>\sqrt{\lambda_{a b}^{b}}$ and $\sqrt{\lambda_{a}}<\sqrt{\lambda_{a b}^{a}}$. Roughly speaking, AI-ab acts more like a balanced scheme in which the large prior inflation of AI-b is reduced and the underinflation of the posterior ensemble given by AI-a is further enhanced. This is consistent not only for $T$ but also for the other variables in CAM4.

\section{Summary}

This paper studies the application of prior and posterior inflation in ensemble Kalman filtering. Spatially 

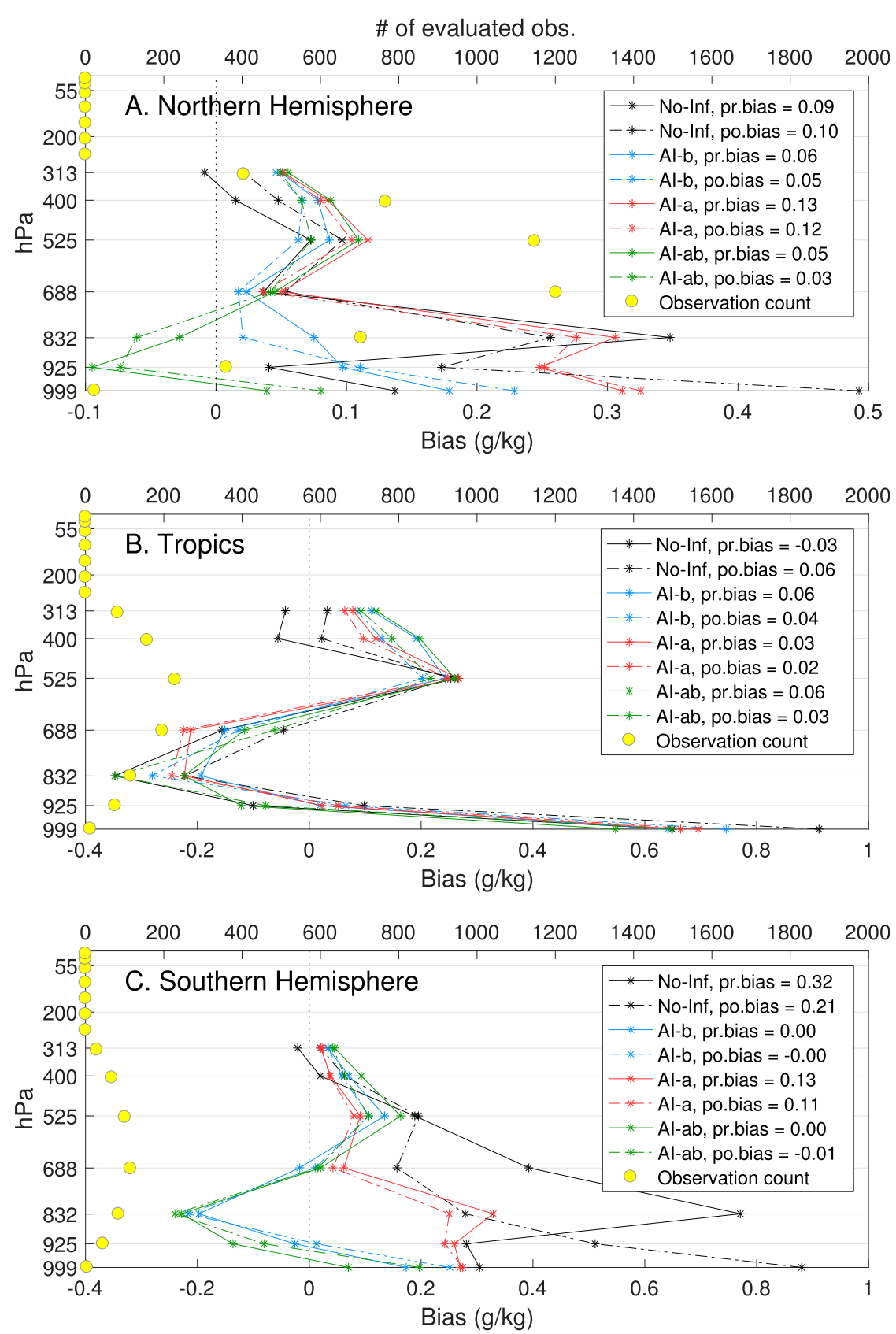

FIG. 9. Profiles of prior (pr.) and posterior (po.) radiosonde specific humidity bias resulting from No-Inf, AI-b, AI-a, and AI-ab DA runs. The computation of the bias is split into three regions: (a) Northern Hemisphere, (b) tropics, and (c) Southern Hemisphere. The numbers in the legend denote the average of the weighted bias over all pressure bins. The filled circles correspond to the temporal-average number of evaluated observations per assimilation cycle.

and temporally varying adaptive prior inflation introduced in A09 is compared to its enhanced variant of E18 using the Community Atmosphere Model assimilating observation sets such as GPS radio occultation refractivity, temperature and wind velocities from radiosondes, ACARS, aircraft, and satellites. The paper also introduces a new adaptive posterior inflation algorithm (AI-a) in addition to a modified and more efficient variant (mAI-a). The modified algorithm features an observation impact removal strategy before updating the posterior inflation distribution the same way prior inflation gets updated in A09 or E18. 

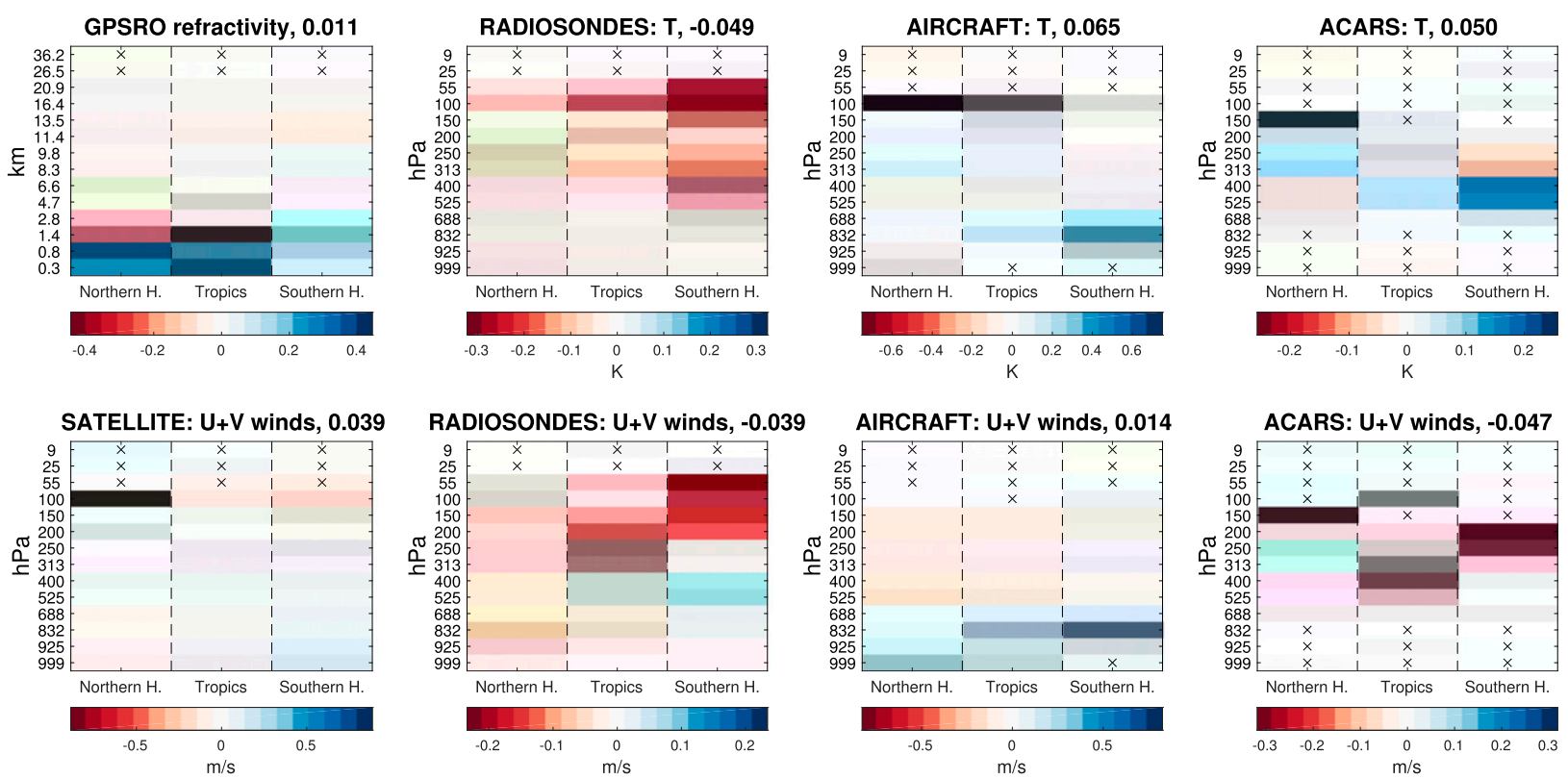

FIG. 10. As in Fig. 5, but comparing the absolute value of the bias resulting from AI-b and AI-ab. Blue (red) color means AI-b's bias is bigger (smaller) than that of AI-ab. A positive overall weighted mean indicates that AI-ab estimates are less biased and vice versa.

CAM4 assimilation results suggest that E18 enhancements are capable of increasing the prediction accuracy given by A09 by almost $5 \%$. In addition, E18 proposes a better match between the ensemble errors and spread.

$\mathrm{AI}-\mathrm{a}$ and $\mathrm{mAI}-\mathrm{a}$ are tested and compared to three other posterior inflation strategies in a simple L63 system. The accuracy generated by AI-a is only marginally higher than that of $\mathrm{mAI}-\mathrm{a}$. Both schemes produce reliable ensemble predictions and perform well compared to already established techniques such as the relaxation to prior spread. Given its computational efficiency, $\mathrm{mAI}-\mathrm{a}$ is adopted, and referred to as AI-a, in all assimilation experiments in this work. Under perfect modeling conditions, AI-a resulted in higher-quality estimates compared to prior inflation (AI-b). This indicated that if sampling errors dominate other error sources in the filter, the proposed posterior inflation can be more useful than prior inflation.

When model errors are dominant, as in the case of CAM4, AI-a was found to be not as useful particularly in the Northern Hemisphere. When compared to AI-b, AI-a suggested inflation values that are almost $50 \%$ smaller. Assimilating a huge number of observations into a limited-size ensemble causes a massive reduction in the ensemble spread, which may not happen in the vicinity of the observations given the biased forecast. The fact that AI-a does not generate large inflation values hinders the filter from restoring enough spread in the analysis ensemble and thus degrades the quality of the estimates in the long term. In regions with sparse observations, AI-a performed quite well and helped remove large percentage of the bias. Compared to RTPS, AI-a resulted in better quality estimates especially for ACARS $T$.

AI-b was consistently better than AI-a at handling model errors. It was found to be more reliable in the sense that the resulting ensemble errors were of the same order as the ensemble spread. Overall the results

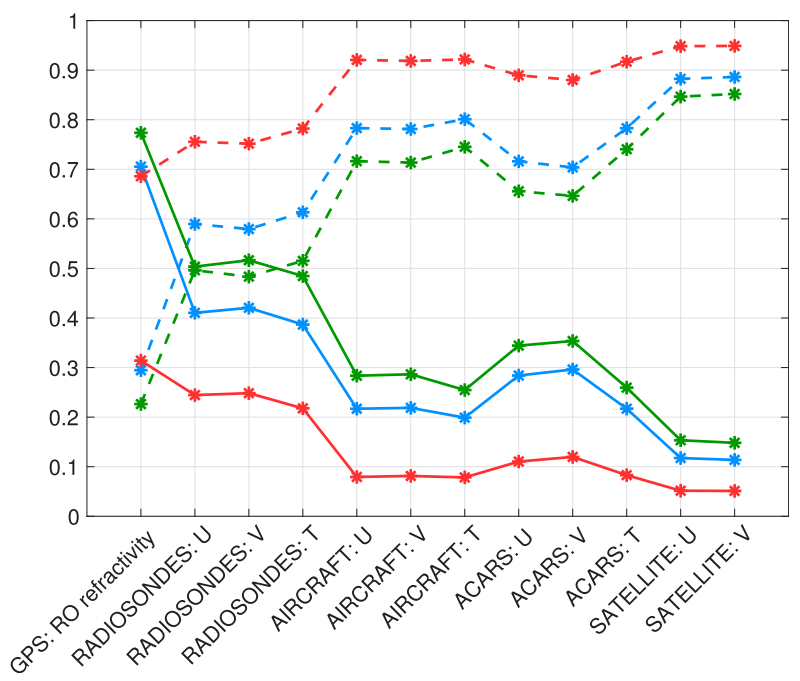

FIG. 11. The information content IC (solid lines) for all assimilated products evaluated for AI-b (blue), AI-a (red), and AI-ab (green). The background content (1-IC) is also shown in dashed lines, as a reference. 

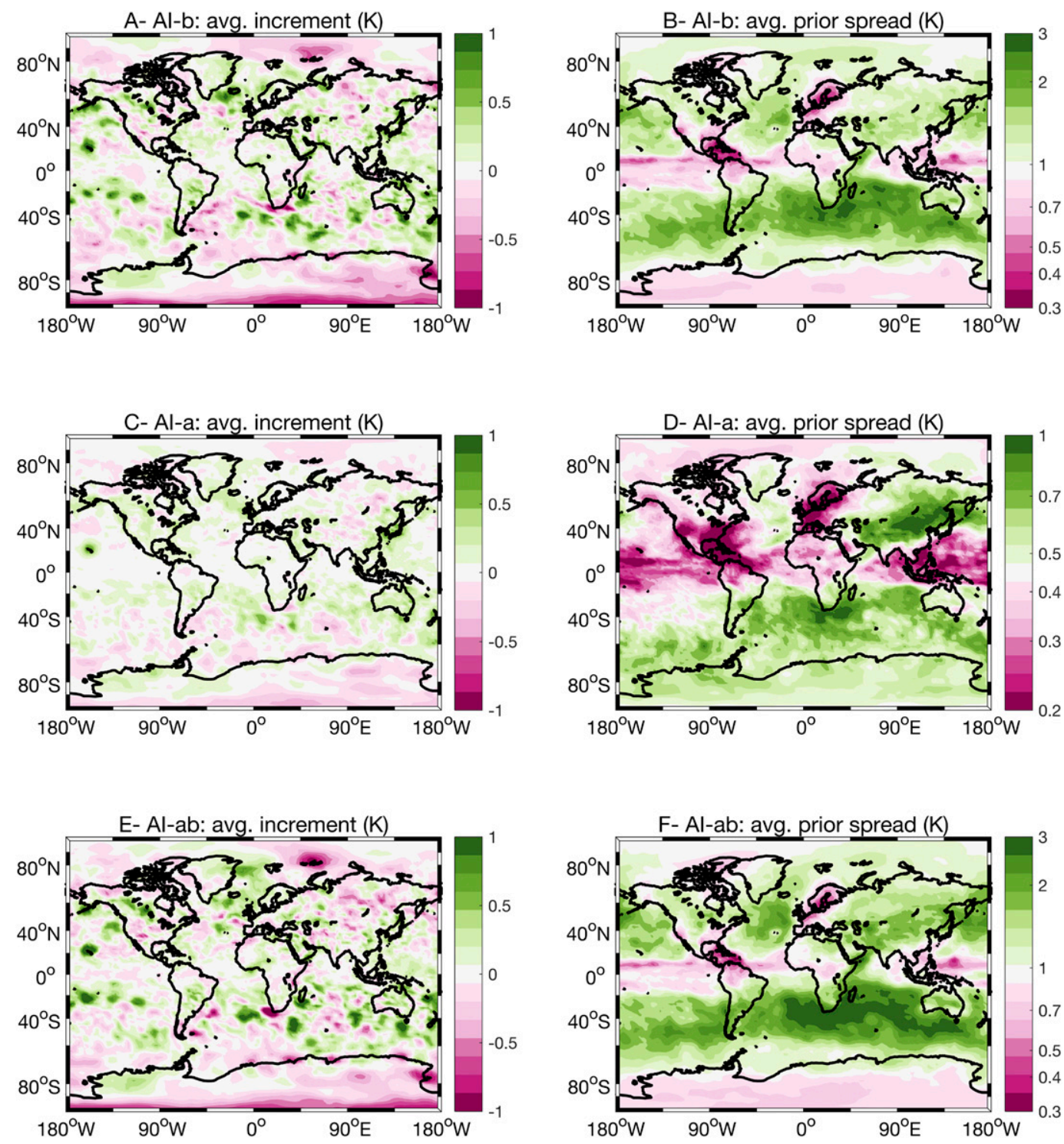

FIG. 12. (left) Average temperature increment at $697 \mathrm{hPa}$, over the last two weeks in September, resulting from AI-b, AI-a, and AI-ab DA runs. (right) Average temperature ensemble spread at the same elevation and during the same period of time. Results from (top) AI-b, (middle) AI-a, and (bottom) AI-ab assimilation runs, respectively.

showed that, in such a biased system, inflating the background statistics before the analysis, which may also be subject to other errors, can help retain sufficient spread for a better assimilation experience. AI-ab assimilation runs, that use both prior and posterior inflation, provided a very compelling performance. Compared to AI-b and AI-a, AI-ab suggested systematic improvements for all variables across the domain. Prior and posterior inflation maps resulting from $\mathrm{AI}-\mathrm{ab}$ were found highly correlated with those obtained using AI-b and AI-a. We also noticed that the magnitude of prior inflation computed in the combination run (AI-ab) is smaller than that obtained using AI-b. In contrast, posterior inflation suggested by AI-ab was larger than that of AI-a. We argue that this feature helps AI-ab yield more stable solutions by minimizing the occurrence of sudden overinflation, from which AI-b suffers.

In summary, combining the enhanced adaptive prior inflation of E18 with the proposed modified posterior inflation algorithm has demonstrated a great potential to enable each inflation component to resolve different filtering issues. CAM assimilation results suggest that prior inflation can help reduce model related deficiencies and posterior inflation is more effective at handling statistical issues such as sampling errors, observation error misspecification, etc. One drawback of 


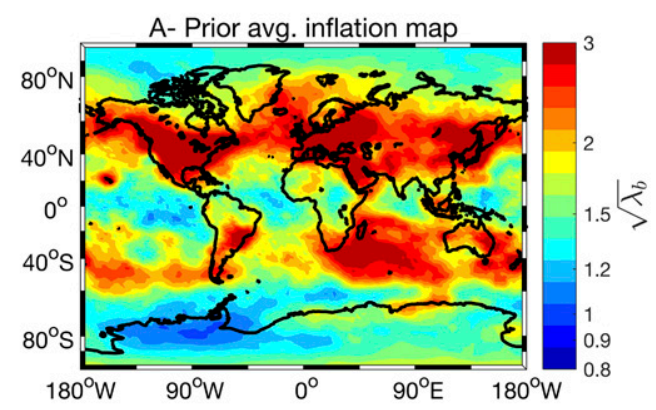

B- Inf. maps time-correlation, Al-b \& Al-ab
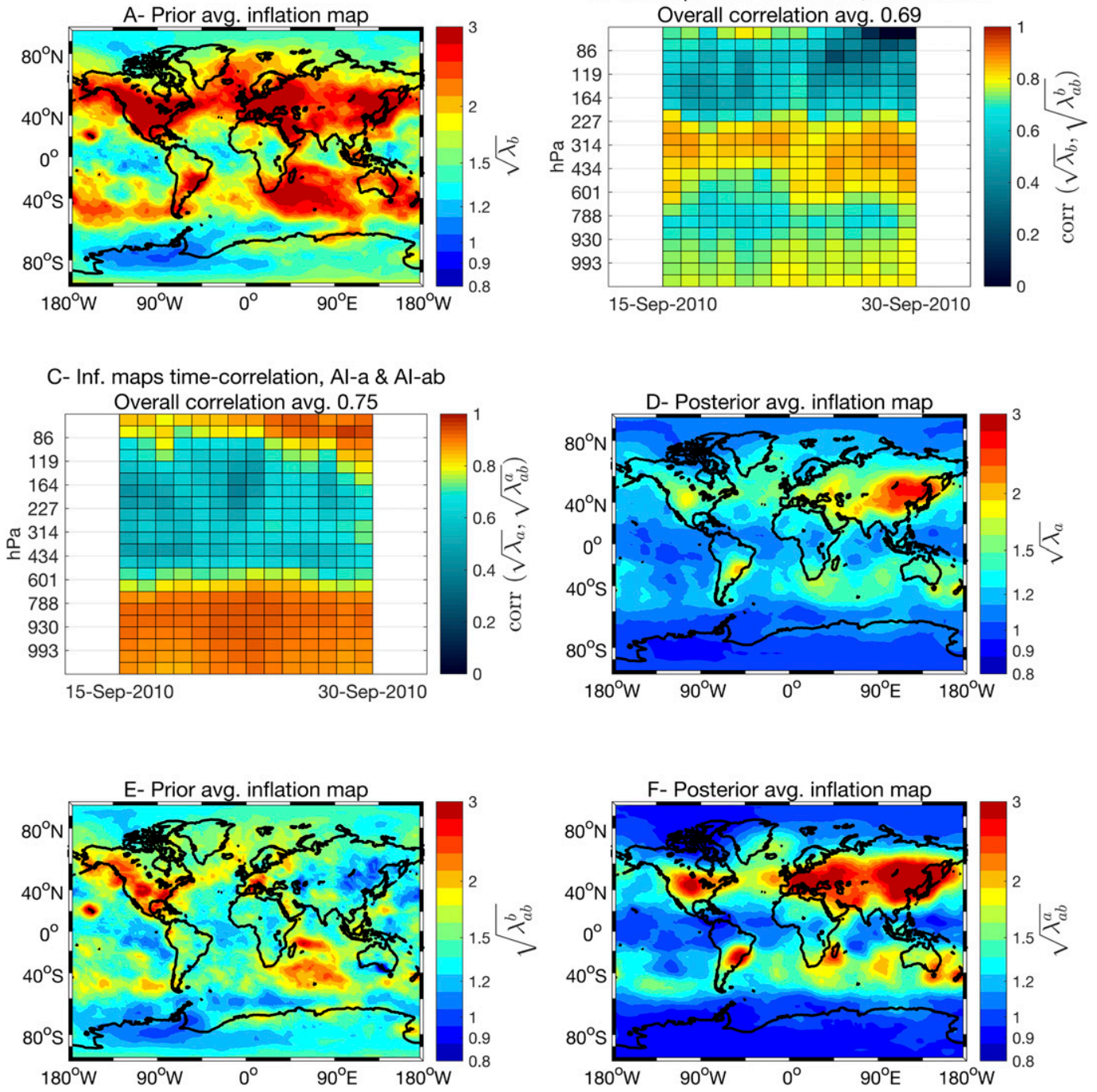

FIG. 13. (top) AI-b: (a) averaged prior inflation $\sqrt{\lambda_{b}}$, and (b) the correlation between $\sqrt{\lambda_{b}}$ and $\sqrt{\lambda_{a b}^{b}}$ by height. (middle) As in (top), but for AI-a,: (c) the correlation between $\sqrt{\lambda_{a}}$ and $\sqrt{\lambda_{a b}^{a}}$ and (d) $\sqrt{\lambda_{a}}$ (posterior). (bottom) $\mathrm{AI}-\mathrm{ab}$ : (e) prior $\sqrt{\lambda_{a b}^{b}}$ and (f) posterior $\sqrt{\lambda_{a b}^{a}}$ average inflation maps.

the current study is that the assimilation experiments are run for only one month, which may be insufficient to draw robust conclusions. In addition, testing these inflation schemes in convective events, such as tropical storms, should give more insights on their usefulness for ensemble-based filters.

Acknowledgments. The authors thank three anonymous reviewers for their helpful comments and suggestions. The first author thanks Craig Bishop, Patrick Raanes, and Moustafa Gharamti for insightful discussions. The authors are grateful for Tim Hoar and Nancy Collins for their technical support with DART. Any opinions, findings, and conclusions or recommendations expressed in this publication are those of the authors and do not necessarily reflect the views of the National Science Foundation.

\section{REFERENCES}

Aksoy, A., D. C. Dowell, and C. Snyder, 2009: A multicase comparative assessment of the ensemble Kalman filter for assimilation of radar observations. Part I: Storm-scale analyses. Mon. Wea. Rev., 137, 1805-1824, https://doi.org/ 10.1175/2008MWR2691.1.

Anderson, J. L., 2003: A local least squares framework for ensemble filtering. Mon. Wea. Rev., 131, 634-642, https:// doi.org/10.1175/1520-0493(2003)131<0634:ALLSFF> 2.0.CO;2.

2009: Spatially and temporally varying adaptive covariance inflation for ensemble filters. Tellus, 61A, 72-83, https://doi.org/ 10.1111/j.1600-0870.2008.00361.x. 
- 2012: Localization and sampling error correction in ensemble Kalman filter data assimilation. Mon. Wea. Rev., 140, 2359-2371, https://doi.org/10.1175/MWR-D-11-00013.1.

—_ and S. L. Anderson, 1999: A Monte Carlo implementation of the nonlinear filtering problem to produce ensemble assimilations and forecasts. Mon. Wea. Rev., 127, 2741-2758, https:// doi.org/10.1175/1520-0493(1999)127<2741:AMCIOT>2.0.CO;2. , T. Hoar, K. Raeder, H. Liu, N. Collins, R. Torn, and A. Avellano, 2009: The Data Assimilation Research Testbed: A community facility. Bull. Amer. Meteor. Soc., 90, 1283-1296, https://doi.org/10.1175/2009BAMS2618.1.

Arakawa, A., and V. R. Lamb, 1981: A potential enstrophy and energy conserving scheme for the shallow water equations. Mon. Wea. Rev., 109, 18-36, https://doi.org/10.1175/15200493(1981)109<0018:APEAEC > 2.0.CO;2.

Bierman, G. J., 1977: Factorization Methods for Discrete Sequential Estimation. Academic Press, 241 pp.

Bocquet, M., 2011: Ensemble Kalman filtering without the intrinsic need for inflation. Nonlinear Processes Geophys., 18, 735-750, https://doi.org/10.5194/npg-18-735-2011.

Cardinali, C., S. Pezzulli, and E. Andersson, 2004: Influence-matrix diagnostic of a data assimilation system. Quart. J. Roy. Meteor. Soc., 130, 2767-2786, https://doi.org/10.1256/qj.03.205.

Cucurull, L., 2010: Improvement in the use of an operational constellation of GPS radio occultation receivers in weather forecasting. Wea. Forecasting, 25, 749-767, https://doi.org/ 10.1175/2009WAF2222302.1.

Dickinson, R. E., K. W. Oleson, G. Bonan, F. Hoffman, P. Thornton, M. Vertenstein, Z.-L. Yang, and X. Zeng, 2006: The Community Land Model and its climate statistics as a component of the Community Climate System Model. J. Climate, 19, 2302-2324, https://doi.org/10.1175/JCLI3742.1.

Dowell, D. C., F. Zhang, L. J. Wicker, C. Snyder, and N. A. Crook, 2004: Wind and temperature retrievals in the 17 May 1981 Arcadia, Oklahoma, supercell: Ensemble Kalman filter experiments. Mon. Wea. Rev., 132, 1982-2005, https://doi.org/10.1175/ 1520-0493(2004)132<1982:WATRIT>2.0.CO;2.

El Gharamti, M., 2018: Enhanced adaptive inflation algorithm for ensemble filters. Mon. Wea. Rev., 146, 623-640, https://doi.org/ 10.1175/MWR-D-17-0187.1.

Evensen, G., 2003: The ensemble Kalman filter: Theoretical formulation and practical implementation. Ocean Dyn., 53, 343-367, https://doi.org/10.1007/s10236-003-0036-9.

Furrer, R., and T. Bengtsson, 2007: Estimation of high-dimensional prior and posterior covariance matrices in Kalman filter variants. J. Multivar. Anal., 98, 227-255, https://doi.org/10.1016/ j.jmva.2006.08.003.

Gaspari, G., and S. E. Cohn, 1999: Construction of correlation functions in two and three dimensions. Quart. J. Roy. Meteor. Soc., 125, 723-757, https://doi.org/10.1002/qj.49712555417.

Gent, P. R., and Coauthors, 2011: The Community Climate System Model version 4. J. Climate, 24, 4973-4991, https://doi.org/ 10.1175/2011JCLI4083.1.

Hodyss, D., and N. Nichols, 2015: The error of representation: Basic understanding. Tellus, 67A, 24822, https://doi.org/ 10.3402/tellusa.v67.24822.

—, W. F. Campbell, and J. S. Whitaker, 2016: Observationdependent posterior inflation for the ensemble Kalman filter. Mon. Wea. Rev., 144, 2667-2684, https://doi.org/10.1175/ MWR-D-15-0329.1.

Hoteit, I., T. Hoar, G. Gopalakrishnan, N. Collins, J. Anderson, B. Cornuelle, A. Köhl, and P. Heimbach, 2013: A MITGCM/ DART ensemble analysis and prediction system with application to the Gulf of Mexico. Dyn. Atmos. Oceans, 63, 1-23, https:// doi.org/10.1016/j.dynatmoce.2013.03.002.

_ D.-T. Pham, M. Gharamti, and X. Luo, 2015: Mitigating observation perturbation sampling errors in the stochastic EnKF. Mon. Wea. Rev., 143, 2918-2936, https://doi.org/ 10.1175/MWR-D-14-00088.1.

Houtekamer, P. L., and H. L. Mitchell, 1998: Data assimilation using an ensemble Kalman filter technique. Mon. Wea. Rev., 126, 796-811, https://doi.org/10.1175/1520-0493(1998) $126<0796$ :DAUAEK $>2.0 . \mathrm{CO} ; 2$.

,-- , and X. Deng, 2009: Model error representation in an operational ensemble Kalman filter. Mon. Wea. Rev., 137, 2126-2143, https://doi.org/10.1175/2008MWR2737.1.

Hurrell, J. W., J. J. Hack, D. Shea, J. M. Caron, and J. Rosinski, 2008: A new sea surface temperature and sea ice boundary dataset for the Community Atmosphere Model. J. Climate, 21, 5145-5153, https://doi.org/10.1175/2008JCLI2292.1.

Jazwinski, A. H., 1970: Stochastic Processes and Filtering Theory. Academic Press, 376 pp.

Kalman, R. E., 1960: A new approach to linear filtering and prediction problems. J. Basic Eng., 82, 35-45, https://doi.org/ 10.1115/1.3662552.

Kistler, R., and Coauthors, 2001: The NCEP-NCAR 50-Year Reanalysis: Monthly means CD-ROM and documentation. Bull. Amer. Meteor. Soc., 82, 247-268, https://doi.org/10.1175/ 1520-0477(2001)082<0247:TNNYRM>2.3.CO;2.

Lauritzen, P. H., A. A. Mirin, J. Truesdale, K. Raeder, J. L. Anderson, J. Bacmeister, and R. B. Neale, 2012: Implementation of new diffusion/filtering operators in the CAM-FV dynamical core. Int. J. High Perform. Comput. Appl., 26, 63-73, https://doi.org/ 10.1177/1094342011410088.

Lin, S.-J., and R. B. Rood, 1996: Multidimensional flux-form semi-Lagrangian transport schemes. Mon. Wea. Rev., 124, 2046-2070, https://doi.org/10.1175/1520-0493(1996)124<2046: MFFSLT $>2.0 . \mathrm{CO} ; 2$.

Lorenz, E. N., 1963: Deterministic nonperiodic flow. J. Atmos. Sci., 20, 130-141, https://doi.org/10.1175/1520-0469(1963) $020<0130: \mathrm{DNF}>2.0 . \mathrm{CO} ; 2$.

Miyoshi, T., 2011: The Gaussian approach to adaptive covariance inflation and its implementation with the local ensemble transform Kalman filter. Mon. Wea. Rev., 139, 1519-1535, https://doi.org/10.1175/2010MWR3570.1.

NCAR/UCAR, 1994: NCEP/NCAR Global Reanalysis Products, 1948-continuing. National Center for Atmospheric Research, Computational and Information Systems Laboratory, Research Data Archive, Boulder CO, accessed 18 May 2018, http:// rda.ucar.edu/datasets/ds090.0/.

_ 2018: The improved Data Assimilation Research TestbedDART Manhattan. NCAR/UCAR, accessed 3 November 2018, https://doi.org/10.5065/D6WQ0202.

Neale, R. B., J. Richter, S. Park, P. H. Lauritzen, S. J. Vavrus, P. J. Rasch, and M. Zhang, 2013: The mean climate of the Community Atmosphere Model (CAM4) in forced SST and fully coupled experiments. J. Climate, 26, 5150-5168, https:// doi.org/10.1175/JCLI-D-12-00236.1.

Oleson, K., and Coauthors, 2008: Improvements to the Community Land Model and their impact on the hydrological cycle. J. Geophys. Res., 113, G01021, https://doi.org/10.1029/ 2007JG000563.

Pham, D. T., 2001: Stochastic methods for sequential data assimilation in strongly nonlinear systems. Mon. Wea. Rev., 129, 1194-1207, https://doi.org/10.1175/1520-0493(2001)129<1194: SMFSDA $>2.0 . \mathrm{CO} ; 2$. 
Raanes, P. N., M. Bocquet, and A. Carrassi, 2019: Adaptive covariance inflation in the ensemble Kalman filter by Gaussian scale mixtures. Quart. J. Roy. Meteor. Soc., 145, 53-75, https:// doi.org/10.1002/qj.3386

Raeder, K., J. L. Anderson, N. Collins, T. J. Hoar, J. E. Kay, P. H. Lauritzen, and R. Pincus, 2012: DART/CAM: An ensemble data assimilation system for CESM atmospheric models. J. Climate, 25, 6304-6317, https://doi.org/10.1175/JCLI-D-11-00395.1.

Sacher, W., and P. Bartello, 2008: Sampling errors in ensemble Kalman filtering. Part I: Theory. Mon. Wea. Rev., 136, 3035-3049, https://doi.org/10.1175/2007MWR2323.1.

Satellite Data Analysis and Archive Center, 2018: The missionEither COSMIC-1/FORMOSAT3 or any other data. University Corporation for Atmospheric Research. UCP, COSMIC Program, Boulder CO, accessed 18 May 2018, http://cdaacwww.cosmic.ucar.edu/.

Wang, X., and C. H. Bishop, 2003: A comparison of breeding and ensemble transform Kalman filter ensemble forecast schemes.
J. Atmos. Sci., 60, 1140-1158, https://doi.org/10.1175/15200469(2003)060<1140:ACOBAE > 2.0.CO;2.

Whitaker, J. S., and T. M. Hamill, 2012: Evaluating methods to account for system errors in ensemble data assimilation. Mon. Wea. Rev., 140, 3078-3089, https://doi.org/10.1175/MWR-D11-00276.1.

- — X. X. Wei, Y. Song, and Z. Toth, 2008: Ensemble data assimilation with the NCEP global forecast system. Mon. Wea. Rev., 136, 463-482, https://doi.org/10.1175/2007MWR2018.1.

Ying, Y., and F. Zhang, 2015: An adaptive covariance relaxation method for ensemble data assimilation. Quart. J. Roy. Meteor. Soc., 141, 2898-2906, https://doi.org/10.1002/ qj. 2576.

Zhang, F., C. Snyder, and J. Sun, 2004: Impacts of initial estimate and observation availability on convective-scale data assimilation with an ensemble Kalman filter. Mon. Wea. Rev., 132, 1238-1253, https://doi.org/10.1175/1520-0493(2004)132<1238: IOIEAO $>2.0 . \mathrm{CO} ; 2$ 